\title{
MAR 271989
}

\section{SMTAG-A Code for the Sequential Analysis of Multiple Tag Gas Releases}

\section{DO NOT MICROFILM COVER}

\section{WuT MicRoFILM COVER}

Prepared for the U.S. Department of Energy Assistant Secretary for Nuclear Energy

\section{(W) Westinghouse}

Hanford Operations and Engineering Contractor for the U.S. Departrnent of Energy under Contract DE-AC06-87RL10930

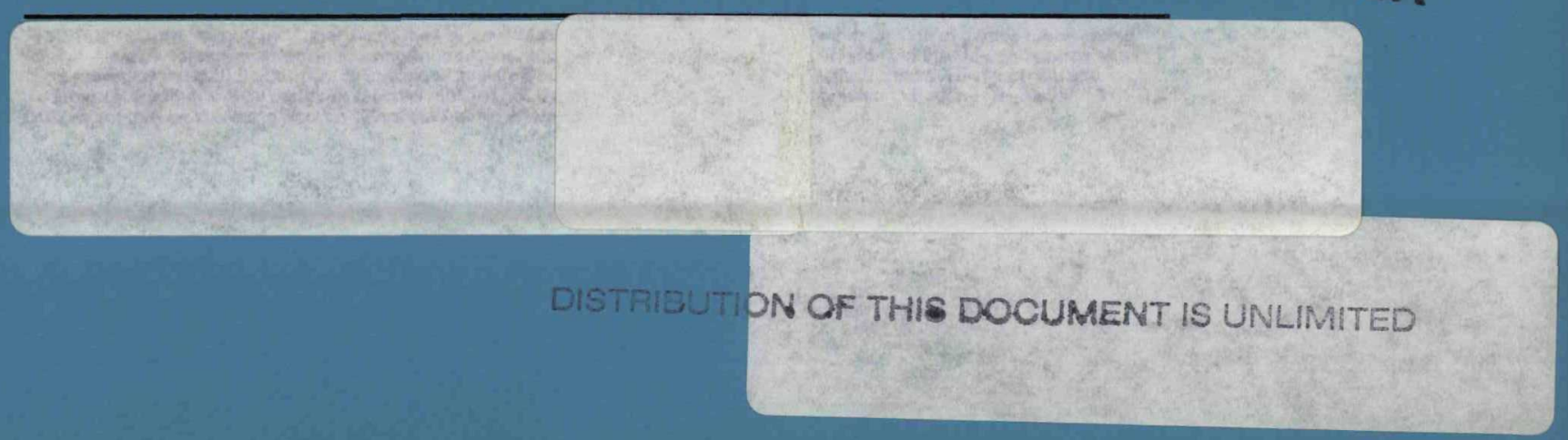




\section{DISCLAIMER}

This report was prepared as an account of work sponsored by an agency of the United States Government. Neither the United States Government nor any agency Thereof, nor any of their employees, makes any warranty, express or implied, or assumes any legal liability or responsibility for the accuracy, completeness, or usefulness of any information, apparatus, product, or process disclosed, or represents that its use would not infringe privately owned rights. Reference herein to any specific commercial product, process, or service by trade name, trademark, manufacturer, or otherwise does not necessarily constitute or imply its endorsement, recommendation, or favoring by the United States Government or any agency thereof. The views and opinions of authors expressed herein do not necessarily state or reflect those of the United States Government or any agency thereof. 


\section{DISCLAIMER}

Portions of this document may be illegible in electronic image products. Images are produced from the best available original document. 


\section{DISCLAIMER}

This report was prepared as an account of work sponsored by an agency of the United States Government. Neither the United States Government nor any agency thereof, nor any of their employees, nor any of their contractors, subcontractors or their employees, makes any warranty, expressed or implied, or assumes any legal liability or responsibility for the accuracy, completeness, or any third party's use or the results of such use of any information, apparatus, product, or process disclosed, or represents that its use would not infringe privately owned rights. Reference herein to any specific commercial product, process, or service by trade name, trademark, manufacturer, or otherwise, does not necessarily constitute or imply its endorsement, recommendation, or favoring by the United States Government or any agency thereof or its contractors or subcontractors. The views and opinions of authors expressed herein do not necessarily state or reflect these of the United States Government or any agency thereof.

\section{Available from}

U.S. Department of Energy

Office of Scientific and Technical Information

Technical Information Center

P.O. Box 62

Oak Ridge, TN 37830

NTIS Price Codes:

Microfiche Copy: $A 01$

Printed Copy:

$\begin{array}{llll}\text { Pages } & \begin{array}{l}\text { Price } \\ \text { Codes }\end{array} & \text { Pages } & \begin{array}{l}\text { Price } \\ \text { Codes }\end{array} \\ 001-025 & \text { A02 } & 301-325 & \text { A14 } \\ 026-050 & \text { A03 } & 326-350 & \text { A15 } \\ 051-075 & \text { A04 } & 351-375 & \text { A16 } \\ 076-100 & \text { A05 } & 376-400 & \text { A17 } \\ 101-125 & \text { A06 } & 401-425 & \text { A18 } \\ 126-150 & \text { A07 } & 426-450 & \text { A19 } \\ 151-175 & \text { A08 } & 451-475 & \text { A20 } \\ 176-200 & \text { A09 } & 476-500 & \text { A21 } \\ 201-225 & \text { A10 } & 501-525 & \text { A22 } \\ 226-250 & \text { A11 } & 526-550 & \text { A23 } \\ 251-275 & \text { A12 } & 551-575 & \text { A24 } \\ 276-300 & \text { A13 } & 576-600 & \text { A25 } \\ & & & \end{array}$

Printed in the United States of America

DISCLM-3.CHP (2-89)

\section{REPRODUCED FROM BEST AVAILABLE COPY}




\section{SMTAG-A Code for the Sequential Analysis of Multiple Tag Gas Releases}

F. A. Schmittroth

Westinghouse Hanford Company

Date Published

January 1989

Prepared for the U.S. Department of Energy Assistant Secretary for Nuclear Energy 
;

$+$ 
WHC-EP-0190

\begin{abstract}
The SMTAG computer code is used to identify breached reactor components that have released tag gas. Using measured isotopic cover-gas concentrations, a ranking of the reactor components most likely to have released tag gas is calculated. The code is specifically designed to analyze sequential gas releases that accompany repeated leaks and/or multiple breaches. Other features include age-tagging, analysis of residual background gases, and estimation of fission-product release factors. A summary of the method, a description of the code, and a users guide are given. Application to an actual case is provided.
\end{abstract}




\section{CONTENTS}

1.0 Introduction . . . . . . . . . . . . . . . . . . 1

2.0 Formalism . . . . . . . . . . . . . . . . . . 2

2.1 Sequential Analysis . . . . . . . . . . . . . . . . 2

2.2 Multiple Component Mixtures . . . . . . . . . . . . 4

2.3 Evaluation of $\mathrm{P}\left(\mathrm{c}_{\mathrm{m}} / \tau\right)$. . . . . . . . . . . . . . . . . . 6

3.0 Code Description . . . . . . . . . . . . . . . . . . . 11

3.1 Main Module . . . . . . . . . . . . . . . . . . 11

3.2 Standard Analysis . . . . . . . . . . . . . . . . . 13

3.3 Sequential Analysis . . . . . . . . . . . . . . . 16

3.4 Probability Integral . . . . . . . . . . . . . . . . . 24

3.5 Auxiliary and Utility Routines . . . . . . . . . . . . 27

4.0 Usage . . . . . . . . . . . . . . . . . . . . . . 31

4.1. General .. . . . . . . . . . . . . . . 31

4.2 Keywords . . . . . . . . . . . . . . . . . . . . 31

4.3 Nonsequential Example . . . . . . . . . . . . . . . 34

4.4 Sequential Example . . . . . . . . . . . . . . . . . 37

4.5 Prob Example . . . . . . . . . . . . . . . . . . . . 38

4.6 Notes . . . . . . . . . . . . . . . . . . . . 39

5.0 References ......................... 41

Appendices

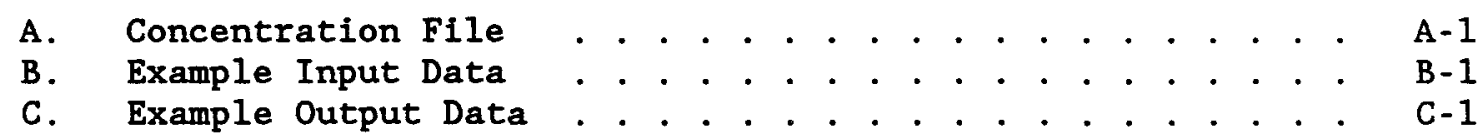




\section{LIST OF FIGURES}

1 An Example of Multiple Measurements $m_{1}$, and $m_{2}$ to Pinpoint Two Leaking Tags in the Presence of Background $b$ Interference. A Nine Tag Design Based on Two Krypton

Ratios is Shown. . . . . . . . . . . . . . . . . . 2

2 Flow Chart for the Subroutine MEASR . . . . . . . . . . . . . 15

3 Flow Chart for the Subroutine PRCALC . . . . . . . . . . . . . . 19

4 Flow Chart for the Subroutine PRCALC (Segment 1) . . . . . . . . 20

5 Flow Chart for the Subroutine PRCALC (Segment 2) . . . . . . . . 22

6 Flow Chart for the Subroutine PRCALC (Segment 3) . . . . . . . . 23

7 Flow Chart for the Subroutine PRCALC (Segment 4) . . . . . . . . 25

8 Flow Chart for the Function PROB . . . . . . . . . . . . . . . . 26

\section{LIST OF TABLES}

1 An Example of System Failure/Leak Propagation from an Initial State $k_{0}$ to a Final State $k_{3}$. . . . . . . . . . . . 4

2 Keywords Used to Control Main Routine . . . . . . . . . . . . 12

3 Scheme for Calculating New Failure Vectors KVP(KP) . . . . . . . 18 


\section{SMTAG -- A CODE FOR THE SEQUENTIAL ANALYSIS OF MULTIPLE TAG GAS RELEASES}

\subsection{INTRODUCTION}

The code SMTAG (Sequential and Multiple TAG Analysis) is used to identify breached reactor components that have released tag gas to the reactor cover gas. Gas tags have been used (Figg et al. 1980 and Lambert 1978) to locate failed fuel pins in both the Fast Flux Test Facility (FFTF) and in the Experimental Breeder Reactor (EBR-II). In the FFTF, other reactor components have been tagged as well, including control assemblies and materials test capsules.

Each component is tagged with a unique isotopic mixture of xenon and/or krypton gas. A mass spectrometer is then used to measure the isotopic composition of a tag release to identify the failed component. A variety of practical issues make it desirable to use a computer code to interpret the isotopic mixture released. Complications include interference from other sources (e.g., gas from previous leakers and air), the possibility of gas releases from more than one component, and the use of duplicate tags.

The need for computer analyses of isotopic releases has long been recognized, and various methods have been proposed (Schmittroth 1973, Figg and Sanchez 1983, Gross 1980). The code Polyfail (Gross 1980) has been successfully used at EBR-II for some time. Based on Bayesian probability methods, another code, TAFLA, (Figg and Sanchez 1983) was developed for use at the FFTF. With experience gained at the FFTF, the need to extend the probabilistic methods was recognized, and a formalism was developed (Schmittroth 1986) to treat not only the possibility of a gas mixture arising from several sources, but to take advantage of the multiple releases that often accompany the breach of a tagged component. This report documents the code SMTAG implementing the extended formalism.

The SMTAG code has been used extensively in gas tag analysis. This has resulted in several code enhancements and has been beneficial in learning to use the code effectively. Supporting information for each analysis is provided that is valuable in ensuring that a correct identification is obtained. The relative amounts of various components in a mixed sample are obtained, including the amount of residual gas from previous leakers, fission-product release-to-birth factors, and xenon-hangup. Statistical tests and other comparisons can flag bad or inconsistent measurements or problems in the supporting nuclear data base.

The formalism for the code is reviewed here in Section 2.0. Details of the code (including descriptions of the main subroutines) are given in Section 3.0. The use of the code is documented in Section 4.0 , along with a discussion of a realistic example. The SMTAG code requires a data base that includes the isotopic amounts of each tag properly corrected for burnup, depletion, and production. The format of this data base is given in Appendix A, but is not otherwise discussed. 


\subsection{FORMALISM}

\subsection{SEQUENTIAL ANALYSIS}

The type of problem for which the code SMTAG was developed is depicted in Figure 1. A very simple nine-tag system based on two distinct krypton ratios is shown. It is assumed that two tags (depicted by the darker dots) are leaking simultaneously and that two measurements are then taken in order to identify the leaking tags. These measurements, taken at different times, reflect the different admixtures of the two tags. A small shift arising from background isotopes (depicted by a "b") in the reactor cover gas is also indicated. A unique identification is not guaranteed, and a Bayesian approach is taken whereby measured information is sequentially added to refine the answer. The basic equations are summarized here. Details may be found in Schmittroth 1986.

The desired knowledge is a complete identification of all failed tags (i.e., failed pins with a specific tag). Since a unique answer may not be possible, the state of knowledge of the system is described by a table of probabilities $P(k)$ that gives the likelihood of each possible state $k$ of the system. The vector $k$ is an NTAG-dimensional vector where each component $k_{i}$ is either 1 or 0 according to whether or not tag $i$ is breached. The total number of tags is NTAG. Note that $k$ denotes a failed state of the system and does not indicate if a particular tag or tags is still leaking. For example $k=(0,1,0,1,0$, then all zeroes) denotes that tags 2 and 4 are failed.

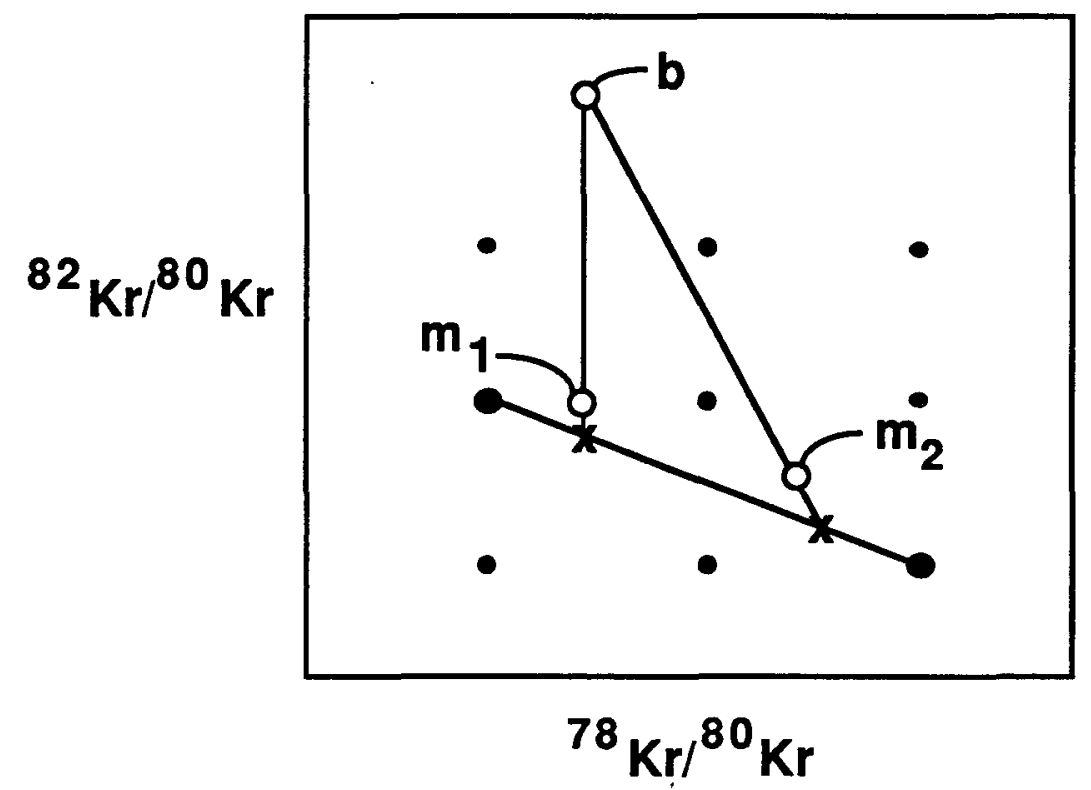

HEDLM 8702-069.1

Figure 1. An Example of Multiple Measurements $\mathrm{m}_{1}$ and $\mathrm{m}_{2}$ to Pinpoint Two Leaking Tags (large dots) in the Presence of Background b Interference. A Nine Tag Design Based on Two Krypton Ratios is Shown. 
A measurement consists of a set of isotopic concentrations $\left\{c_{m i}\right\}$ that may well include fission product isotopes as well as tag isotopes. This measurement is then used to update a set of probabilities $\{P(k)\}$ that describes the system before the measurement to obtain a new set of probabilities $\left\{\mathrm{P}^{\prime}\left(\mathrm{k}^{\prime} / \mathrm{c}_{\mathrm{m}}\right)\right\}$ that describes the system after the measurement:

$$
P^{\prime}\left(k^{\prime} / c_{m}\right)=\sum_{k} P(k) T\left(k \rightarrow k^{\prime}\right) P\left(c_{m} / k \rightarrow k^{\prime}\right)
$$

where

$$
P\left(c_{m} / k \rightarrow k^{\prime}\right)=\sum_{\tau \in\left(k \rightarrow k^{\prime}\right)} P\left(c_{m} / \tau\right) P\left(\tau / k \rightarrow k^{\prime}\right)
$$

The transition matrix $T\left(k \rightarrow k^{\prime}\right)$ gives the probability that the state $k$ evolves to state $k^{\prime}$ during the time of the measurements and depends on the assumed a priori failure probabilities for the various tags. Results of the measurement are included in $P\left(c_{m} / k \rightarrow k^{\prime}\right)$, the conditional probability that the measured values $c_{m}$ are obtained when the system goes from state $k$ to state ' $k^{\prime}$. The different gas releases that may occur in the $k \rightarrow k^{\prime}$ transition are called channels. Consider the simple case where the initial and final states are the same and are defined by both tags 2 and 4 in the failed state. For this single transition, three different channels of gas escape are possible corresponding to efther tag 2 or 4 leaking or both leaking. These possibilities are included in the $r$-sum in Equation 2 where $P\left(c_{m} / \tau\right)$ gives the probability that the measured concentrations $c_{m}$ are obtained from channel $r$ of the $k \rightarrow k^{\prime}$ transition. The probability of this channel occurring is given by $P\left(\tau / k \rightarrow k^{\prime}\right)$.

A straightforward calculation of all the possible final probabilities $\mathrm{P}^{\prime}\left(\mathrm{k}^{\prime} / \mathrm{c}_{\mathrm{m}}\right)$ is not possible. Assume that the system consists of NTAG - 100 distinct tags. Then since each tag may be falled or not, the total number of possible system states is $2^{100} \approx 1.3 \times 10^{30}$. The transition matrix $\mathrm{T}\left(\mathrm{k} \rightarrow \mathrm{k}^{\prime}\right)$ could potentially be a $2^{100} \times 2^{100}$ matrix. The resolution of this problem is to calculate the terms and sums in Equations 1 and 2 as they would arise from actual system behavior.

Initially one starts with a system where there are no failures: $P(k-0)=1$, and $P(k)=0$ for all states $k$, where $k$ represents one or more failed tags. The propagation to a final state $\mathrm{k}^{\prime}$ is then limited to those states that can be reached by a single or double leak/failure. Thus, state $k^{\prime}$ can contain at most two more failed tags than state $k$. Of course as sequential measurements are added, the number of failed tags is limited only by the number of sequential steps and the total number of tags.

A simple example should help clarify these definitions and ideas. Table 1 shows the propagation of the system from an initial unfailed state $k_{0}$ to a state $k_{3}$ through a succession of leaks and failures. First pin 2 fails with an a priori probability of $\pi_{2}$. For this transition, there is only a single channel. Next, pin 1 fails with an a priori probability 
of $\pi_{1}$. At the same time, pin 2 continues to leak (one possible channel for this transition). The other possible channel belonging to this transition is that pin 2 does not continue to leak. Finally, in the last step, no new pins fail, pin 1 continues to leak, and pin 2 stops leaking. In this example, the channel probabilities $\gamma_{1}$ and $\gamma_{2}$ are equal to the respective $a$ priori probabilities for pins 1 and 2 to leak, given that they have already failed. These results are approximate and assume that the a priori failure probabilities and a priori leak probabilities are small. Note the $k_{2} \rightarrow k_{3}$ transition could occur in two other ways: Tag 2 could leak with and without tag 1 leaking.

Table 1. An Example of System Failure/Leak Progagation from an Initial state $k_{0}$ to a Final state $k_{3}$.

\begin{tabular}{|c|c|c|c|c|c|c|c|}
\hline \multirow[b]{2}{*}{ Tag (pin) } & \multicolumn{7}{|c|}{ Failure vectors } \\
\hline & $\mathbf{k}_{0}$ & & $\mathbf{k}_{1}$ & & $\mathbf{k}_{2}$ & & $\mathbf{k}_{3}$ \\
\hline 1 & 0 & & 0 & $\rightarrow$ & 1 & $\rightarrow$ & 1 \\
\hline 2 & 0 & $\rightarrow$ & 1 & $\rightarrow$ & 1 & & 1 \\
\hline 3 & 0 & & 0 & & 0 & & 0 \\
\hline$T\left(k \rightarrow k^{\prime}\right)$ & & $\pi_{2}$ & & $\pi_{1}$ & & 1 & \\
\hline$P\left(\tau / k \rightarrow k^{\prime}\right)$ & & 1 & & $\gamma_{2}$ & & $\gamma_{1}$ & \\
\hline
\end{tabular}

\subsection{MULTIPLE COMPONENT MIXTURES}

In order to obtain $\mathrm{P}\left(\mathrm{c}_{\mathrm{m}} / \mathbf{k} \rightarrow \mathrm{k}^{\prime}\right)$, it is necessary to calculate the cover gas concentrations that arise when the system goes from state $k$ to state $k^{\prime}$. These calculated concentrations can consist of a mixture of several components including multiple leakers and background gas already in the cover gas.

The combined cover gas concentration is thus given by

$$
c_{\tau i}(q, \theta)=\theta \sum_{\alpha} q_{\alpha}^{p} a_{\alpha i}^{\tau}+\sum_{\beta} q_{\beta}^{b} c_{\beta i}^{b}
$$

where the two terms represent gas leaking to the cover gas and background already in the cover gas.

In the first term, the absolute amount of isotope $i$ in component $\alpha$ 
available to leak is denoted by $a_{\alpha i}^{\tau}$. For each component $\alpha$, these values are multiplied by the fraction $q_{\alpha}^{p}$ that actually leaks. Next, the sum over all leaking components is completed and multiplied by the conversion factor $\theta$. This conversion factor between the cover gas concentration and the amount of gas leaking to the cover gas depends on various reactor parameters, such as the cover gas volume and the purge rate. In the code, the conversion factor is adjusted based on the actual measured concentrations. The superscript $\tau$ denotes which channel is being considered (i.e., what particular set of reactor components is assumed to be leaking).

In a similar fashion, the background term consists of a sum of various components that the user estimates to be part of background cover gas. The factor $\theta$ is not included, since the cover gas concentrations $\{c \not i\}$ are specified directly and not the absolute amount of gas in the cover gas. The interpretation of the leak fractions $\left\{q_{\beta}\right\}$ is also slightly different for the background terms; they represent adjustable corrections to the concentrations specified by the user.

A leaking reactor component (pin) can give rise to several components in Equation 3, organized according to the following hierarchy. A fuel pin, for example, releases both tag and fission-product isotopes. These are maintained as separate components in Equation 3, each with its own corresponding $q_{\alpha}$ so that one can account for different leak fractions for the tag and fission-product isotopes. Next, each of these components consists of a xenon component and a krypton component. Again, this organization allows distinct leak-fractions for xenon and krypton.

For background components, the distinction between tag and fissionproduct isotopes is not maintained, because the source is no longer identifiable. However, separate xenon and krypton components are still included. In general, the fractions of xenon and krypton that leak from fuel pins are very similar; however, for background components noticeable differences are seen. Specifically, xenon gas tends to be detected for longer periods than krypton.

A convenient shorthand for Equation 3 is

$$
c_{\tau}(\mathbf{q}, \theta)=A_{\tau}(\theta) q
$$

where the A matrix is defined by inspection.

In practice, all pertinent isotopes are included on an equal basis including both fission-product and tag isotopes. In this way, the use of techniques such as age-tagging (identification based on gas isotope burnin or burnout that is dependent on neutron fluence) is handled automatically and on a consistent basis with the rest of the analysis. 


\subsection{EVALUATION OF $P\left(c_{m} / r\right)$}

For the gas release accompanying the channel/transition $r$, the probability of measuring the concentrations $c_{m}$ is given by

$$
P\left(c_{m} / \tau\right)=\frac{\eta_{c} \eta_{q}}{L} \int_{0}^{L} d \theta \int d q\left\{e^{-\frac{1}{2} Q^{2}(q, \theta)}\right\}
$$

where

$$
Q^{2}(q, \theta)=\left\|q-q_{0}\right\|_{q}^{2}+\left\|c_{m}-c_{\tau}(q, \theta)\right\|_{c}^{2}
$$

The second term is a measure of how closely the calculated concentrations match the measured concentrations for a given set of leak fractions $\left(q_{\alpha}\right)$ and is given by

$$
\left\|c_{m}-c_{\tau}\right\|_{c}^{2}=\left(c_{m}-c_{\tau}\right)^{t} M_{c}^{-1}\left(c_{m}-c_{r}\right)
$$

where $M_{c}$ is the covariance matrix for the measurement uncertainties.

These uncertainties are assumed to be uncorrelated and $M_{c}$ is diagonal with diagonal elements equal to the square of the standard deviations for the measurements.

The first term constrains the leak fraction $q$ to be near the fixed values $q_{0}$ in the sense of the covariance matrix $\mathrm{M}_{\mathrm{q}}$ :

$$
\left\|q-q_{0}\right\|_{q}^{2}=\left(q-q_{0}\right)^{t} M_{q}^{-1}\left(q-q_{0}\right)
$$

(Note that the important contributions to the integral occur when $\mathrm{Q}^{2}$ is small.)

For the nonbackground components, the role of $q_{0}$ is to provide a weak constraint on the value of $q$ in circumstances where the measured concentrations do not dictate the leak fractions through the second term in Equation 6. For both fission-product and tag components, qoa is set to 0.5 with a standard deviation of 0.5 , roughly reflecting that the leak fractions are between 0 and 1 . A common value for the tag and fission-product components weakly implies a fission-product release to birth factor of unity. Because of the conversion factor $\theta$, only the relative values are significant. For background components, both $q_{0}$ and its standard deviation are specified by the user.

As with the $M_{c}$ covariance matrix, the diagonal elements of the 
$\mathrm{q}_{0}$-covariance matrix $\mathrm{M}_{\mathrm{q}}$ are set to the square of the respective standard deviations. However, non-zero off-diagonal elements may be specified to correlate the xenon and krypton leak fractions. Presently correlations may be separately specified for background and nonbackground components. The intent of these a priori correlations is to constrain the amount of krypton relative to the amount of xenon. Because the desired constraint is a ratio and the code treats absolute leak fractions, the algorithm is rather weak. A logarithmic algorithm constraining the ratio might be more effective.

The constants are normalization values for Gaussian probability density functions

$$
\eta_{c}=\sqrt{\frac{\operatorname{det}\left|M_{c}^{-1}\right|}{(2 \pi)^{n_{c}}}}
$$

and

$$
\eta_{\mathrm{q}}=\sqrt{\frac{\operatorname{det}\left|\mathrm{M}_{\mathrm{q}}^{-1}\right|}{(2 \pi)^{n_{\mathrm{q}}}}}
$$

the constant $\mathrm{L}$ is any large number.

The integral in Equation 5 is approximated, in part, by evaluating the integrand at its peak value where $Q^{2}$ is a minimum (Schmittroth 1986). The result is

$$
\mathrm{P}\left(c_{\mathrm{m}} / \tau\right)=\left(\frac{\eta_{\mathrm{c}} \eta_{\mathrm{q}}}{\eta_{\alpha} \eta_{\mathrm{Q}}}\right)\left(\frac{\theta_{0} \alpha^{\mathrm{n}_{\mathrm{q}}-2}}{\mathrm{~L}}\right) \mathrm{e}^{-\frac{1 / 2}{\mathrm{Q}_{\mathrm{min}}^{2}}}
$$

where

$$
Q_{\min }^{2}=Q^{2}\left(\alpha q_{m i n}, \theta_{0}\right)
$$

and $q_{\min }\left(\theta_{0}\right)$ are the leak fractions that minimize $Q^{2}\left(q, \theta_{0}\right)$ for a given value for the conversion factor $\theta_{0}$.

The components of $q_{\text {min }}$ have a direct physical interpretation as the fractional amounts of each component of the mixture that are most consistent with the measured concentrations. As such, the components of $q_{m i n}$ are very important in interpreting the results of the code. These components 
can give a direct measure of the amount of one tag in the mixture relative to a second tag, the amount of krypton relative to the amount of xenon, fission-product to tag isotope release fractions, and the importance and size of various postulated background components.

The algorithm used to solve the least-squares problem for $q_{m i n}$ is

$$
q_{\min }\left(\theta_{0}\right)=q_{0}+M_{Q} A_{\tau}^{t}\left(\theta_{0}\right) M_{c}^{-1}\left[c_{m}-A_{\tau}\left(\theta_{0}\right) q_{0}\right]
$$

where $A_{T}\left(\theta_{0}\right)$ is via Equation 4.

The covariance matrix $\mathrm{M}_{0}$ gives the uncertainties and correlations for the final leak fractions as determined by both the initial constraints specified by $\mathrm{M}_{\mathrm{q}}$ and the uncertainties in the measured concentrations and is given by

$$
M_{Q}^{-1}=M_{q}^{-1}+A_{\tau}^{t}\left(\theta_{0}\right) M_{c}^{-1} A_{\tau}(\theta)
$$

The standard deviations of the estimated leak fractions $\left(q_{\mathrm{min}, \alpha}\right)$ are given by the square roots of the diagonal elements of $\mathrm{M}_{\mathrm{Q}}$ and are also important in interpreting the results of the code. If the standard deviations are markedly smaller than the corresponding initial uncertainties specified in $\mathrm{M}_{\mathrm{q}}$, then the user knows that these components are strongly determined by the measurements. A negligible reduction in the uncertainty for a particular leak fraction implies that al though the component was included in the analysis, little is learned of its actual size from the measurements. In Equation 10, $\eta_{Q}$ is defined by Equation $9 b$, with $\mathrm{M}_{\mathrm{q}}$ replaced by $\mathrm{M}_{\mathrm{Q}}$.

Some physical interpretation for Equation 12 is apparent. Final estimated leak fractions $q_{m i n}$ are given as the initial estimates corrected by a factor that is proportional to the difference $\left(c_{m}-c_{\tau}\right)$ between the measured and calculated concentrations.

Introduction of the a priori leak fractions $\left\{q_{o \alpha}\right\}$ is somewhat artificial but provides a simple solution to an otherwise difficult problem. In some situations and for a known set of leaking components, a single set of measured concentrations can uniquely fix the fraction of each component that is leaking. Generally, a measurement of $n$ distinct tag ratios will uniquely determine the fractional amounts leaked by $n+1$ leaking components . In other situations, however, the leak fractions $\left\{q_{\alpha}\right\}$ are indeterminate, and a standard least-squares approach will fail. By using a priori leak fractions with large uncertainties, the least-squares method applies equally well in both the indeterminate and overdetermined examples. For the overdetermined cases, the leak fractions are fixed by the measured concentrations, and the effect of the a priori values is minimal; in cases where the leak fractions are poorly determined, both the final estimated values and their respective uncertainties remain near the a priori values. Any individual case may contain a mixture where some components are well 
determined and others are poorly determined.

The conversion factor $\theta$ is estimated as follows. Since the measured concentrations are insufficient to distinguish between a change in $\theta$ and a change in the total fraction of gas that leaks, the a priori leak fractions $\left(q_{\alpha O}\right)$ are also used to estimate $\theta$. Specifically, $\theta$ is adjusted to minimize the difference between the calculated and measured concentrations, where the calculated concentrations are based on the a priori leak fractions. The resulting equation is

$$
\theta_{0}=\frac{\left(A_{\tau} q_{o}^{P}\right)^{t} M_{c}^{-1}\left(c_{m}-c_{b} q_{o}^{b}\right)}{\left(A_{\tau} q^{P}\right)^{t} M_{0}^{-1}\left(A_{\tau} q_{c}^{P}\right)}
$$

The variable $\alpha$ in Equations 10 and 11 gives a correction to this conversion factor

$$
\hat{\theta}=\frac{1}{\alpha} \theta_{0}
$$

and is given by (see Schmittroth 1986)

$$
\alpha=\sigma_{\alpha}^{2}\left(q_{\min }^{\prime t} M_{q}^{-1} q_{o}^{\prime}\right)^{-1}
$$

where

$$
\sigma_{\alpha}^{2}=\left(q_{\min }^{\prime t} M_{q}^{-1} q_{\min }^{\prime}\right)^{-1}
$$

The primes denote that only nonbackground components are included in the calculation, an improved approximation over the corresponding equation in Schmittroth (1986). [The variable $\alpha$ is denoted by $\xi$ in Schmittroth (1986)]. Another slight difference compared with Schmittroth (1986) is the exponent of $\alpha$ in Equation 10, a consequence of both a slightly different approximation and the correction of an error in Schmittroth (1986).

The total minimum quadratic form $Q_{m}^{2}$ in is denoted by chi-square and is output for all likely candidate leaker combinations. It gives a direct indication of how consistent each potential combination of leakers is with the measured concentrations and is an excellent diagnostic tool. As shown in Equation 6, this overall chi-square is broken down into two terms. The first term displays the consistency between the a priori leak fractions and the leak fractions obtained in the least-squares analysis. The second term shows the agreement between the calculated and measured concentrations. 
Because $M_{c}$ is a diagonal matrix, the chi-squared term for the concentrations can be further decomposed giving the agreement with measured concentrations on an isotope-by-isotope basis

$$
\left\|c_{m}-c_{\tau}\right\|_{c}^{2}=\sum_{i=1}^{n_{c}} \frac{1}{\sigma_{c i}^{2}}\left(c_{m i}-c_{\tau i}\right)^{2} .
$$

The final leak-fraction covariançe matrix is not diagonal; nevertheless, the terms $\left(\left(\mathrm{q}_{\mathrm{min}}, \alpha^{-\mathrm{q}_{o \alpha}}\right) / \sigma_{\mathrm{q} \alpha}\right\}$, where $\left\{\sigma_{\mathrm{q} \alpha}{ }^{2}\right\}$ are the diagonal elements, are usually a reliable indicator of inconsistencies and are output. For example, if a background component in the cover gas is much larger than that estimated by the user, it would be flagged. Note, however, that these terms do not add to the total leak-fraction residual $\left\|q_{\min }-q_{0}\right\|_{q}$. 
WHC-EP-0190

\subsection{CODE DESCRIPTION}

\subsection{MAIN MODULE (MTAG)}

The main module is MTAG, that controls program execution by reading keywords and then calling appropriate subroutines. A brief summary of available keywords and their actions is given in Table 2. The simplest possible nontrivial runstream consists of the keywords MEASUREMENT and STOP. Reading of the keyword MEASUREMENT causes MTAG to call the subroutine MEASR, that reads a set of measured isotopic concentrations and calls the appropriate routines to complete the analysis as described in Section 3.2 .

The runstream is called sequential if it includes more than one MEASUREMENT keyword. Sequential runs can also be made by storing the results of one run on Unit 11 with the SAVE keyword. The results can be read on Unit 11 by a subsequent run with the first keyword SEQUENTIAL. Results using a SEQUENTIAL keyword are the same as if the two runstreams were merged into a single run.

The first action by the code is to check if the first keyword is SEQUENTIAL. If it is, system parameters are read from Unit 11 . These parameters include the NK failure vectors $(\mathrm{KV}(\mathrm{N}))$, and associated failure probabilities ( PR(N) \} obtained from the previous measurement analysis. Note here that the failure vector $\mathrm{KV}$ and the associated failure probability $P R$ are indexed by a common array index $N$ in contrast to the notation $P(k)$ introduced in Section 2.1. Also included are: a priori fallure probabilities (SPI(I) \}, a priori leak probabilities ( GAM(I) \}, previously specified background components, and the specification of assured leakers. These values are all included in the labeled common blocks :

COMMON /SYSCOM/ NK, PR(NK0), KV(NK0), KS(10), NLKRS, NBKG, 1 $\mathrm{CB}(\mathrm{NIO}, 5), \operatorname{SIGB}(5), \operatorname{GAM}(\mathrm{NTAGO})$, SPI (NTAGO)

COMMON /SYACOM/ BKLABL(5), LSTMSR . 
Table 2 Keywords Used to Control Main Routine.

\begin{tabular}{|c|c|c|}
\hline Keyword & Subroutine & Purpose \\
\hline SEQUENTIAL & - & Read system parameters from Unit 11 . \\
\hline ITYPE & SETTYP & $\begin{array}{l}\text { Select ITYPEs to be read from } \\
\text { concentration file. }\end{array}$ \\
\hline MEASUREMENT & MEASR & $\begin{array}{l}\text { Read measured concentrations and begin } \\
\text { standard execution. }\end{array}$ \\
\hline PROBABILITY & PROB & $\begin{array}{l}\text { Analyze a single specified leaker } \\
\text { combination. }\end{array}$ \\
\hline SAVE & - & Save system parameters to Unit 11 . \\
\hline BACKGROUND & BKGRND & Enter background. \\
\hline POOR & FLGBAD & Selected isotopes not used in analysis. \\
\hline LEAKER & LEAKER & Selected tags are assured leakers. \\
\hline UNCERTAINTY & UNCER & Reset measurement uncertainties. \\
\hline CORRELATION & XKCORR & Reset xenon-krypton amounts correlation. \\
\hline PCUT & - & $\begin{array}{l}\text { Reset cutoff probability of analyzed } \\
\text { combinations. }\end{array}$ \\
\hline PRINT & PRNT & Print options. \\
\hline STOP & - & Terminate analysis. \\
\hline
\end{tabular}


The NLKRS-assured leakers are defined by the KS-array, and data for the NBKG background components are given in the arrays CB, SIGB, and BKLABL. One should note that these variables, and only these variables, are passed from one run to another via Unit 11. Other values, such as reset uncertainties are not included.

The next action is a call to LOADG to read the isotopic amounts defining the gas tags from the concentration file (Unit 10). It is assumed that all values on the concentration file have been corrected for isotopic depletion and production.

Both tag and fission-product isotopes are maintained separately on the concentration file, and the gas amounts are read into the arrays G(NI, NTAG), and GFP(NI, NTAG), respectively. The tag order and the total number of tags NTAG are determined from the concentration file. This requires some caution when the SEQUENTIAL keyword is used. If a new concentration file with a different tag order were used for the subsequent run, the values for SPI and GAM would become scrambled.

A variable named ITYPE(NTAG) is also maintained for each tag on the concentration file. The keyword ITYPE allows the user to select only those tags belonging to a specified set of ITYPEs (e.g., MOTA tags), to be read from the concentration file. In this way, the analysis may be shortened considerably. This keyword must follow the SEQUENTIAL keyword when it exists.

After the concentration file has been loaded so that the total number of tags NTAG has been specified, a call is made to INITL to initialize the a priori failure and leak probabilities SPI and GAM (if they were not previously set by the use of a SEQUENTIAL keyword). The number NK of failure vectors, the number NKBG of background components, and the number NLKRS of assured leakers are also set to zero by INITL.

After these initialization steps, the code checks for other keywords given in Table 2. A MEASUREMENT keyword may be used to initiate an analysis or first, other parameter setting routines may be called, (e.g., background specifications). In most cases, a natural order for keywords is used. For instance, if an analysis initiated by a MEASUREMENT keyword required revised uncertainties, then the UNCERTAINTY keyword along with the revised uncertainties must precede the MEASUREMENT keyword. A second UNCERTAINTY keyword could then be used to revise the uncertainties for further analyses.

\subsection{STANDARD ANALYSIS (MEASR)}

Each MEASUREMENT keyword results in a call to the subroutine MEASR to obtain an updated (primed) set of failure vectors $\{\mathrm{KVP}(\mathrm{N})\}$ and probabilities \{ PRP(N) | via Equations 1-2.

As noted earlier, it is impossible to consider all possible failure states of the system. Instead, one starts by assuming that the only state of the system with a non-zero probability is where all tags are intact. The total number NK of failure vectors considered by the system is set to one. 
WHC-EP-0190

The vector $\mathrm{KV}(1)$ is set to zero to indicate that there are no failed tags, and the associated probability PR(1) is set to one. (The variable NK is initially set to zero as a flag for this situation.) Additional failure vectors are created by the consideration of all possible single and double failures.

The vector $K V(N)$ specifies the Nth failure state considered by the code. In the code, each element of the array $\mathrm{KV}(\mathrm{N})$ is actually an integer array with four dimensions. In other words, $\mathrm{KV}(\mathrm{N})$ is really represented by $\mathrm{KV}(I, N),(I=1,4)$. For a machine with 63 bits (not including the sign bit) in an integer, each element of $\mathrm{KV}(\mathrm{N})$ thus contains $4 \times 63=252$ bits and can represent the failure state of 252 distinct tags. A failed tag is designated by setting the proper bit to one. Thus, KV(1) $=0$ is shorthand for $[\mathrm{KV}(I, 1)-0, I=1,4]$ and denotes that all tags in State 1 are intact. A UNIVAC version of the code uses a CHARACTER representation for $\mathrm{KV}(\mathrm{N})$ and machine-dependent bit-setting functions.

A schematic flowchart of MEASR is given in Figure 2. A call is first made to RDCON to read in the measured isotopic concentrations. Next, BSUMRY is called to provide a printed summary of the current background values. A call to SIGSET then revises the uncertainties for the measured concentrations. For example, uncertainties are increased for isotopes with very small concentrations. After ISWTCH is set to zero, PRCALC is then called to obtain the desired probabilities. The subroutine PRCALC performs the calculations indicated in Equations 1-2 and in turn calls PROB to evaluate the probability integrals $P\left(c_{m} / k \rightarrow k^{\prime}\right)$. The variable ISWTCH is a flag to control the values output from the function PROB and is discussed later.

There are two parts to the remainder of MEASR, truncation of the 1ist of failure vectors and final sorting and output. Truncation of the failure vectors is useful in presenting the results, but it is required to obtain manageable results in sequential runs. In sequential runs without truncation, the successive addition of single and double failures to previous failure vectors quickly exceeds the computer's capacity.

Before truncation, a check (NLP $>0$ ) is made to see if any failure vectors with non-zero probabilities were returned by the call to PRCALC. If there were, the sum of the probabilities is normalized to one and truncation proceeds. Truncation is repeated until the new number NKP of failure vectors is less than NKO - 100 and the number NFL of failed tags is less than 35. These values were chosen as a compromise between maintaining a manageable list size and not discarding potential but low probability failures. Truncation is obtained by repeated calls to FINDRD that does a binary search to obtain an appropriate reduction factor REDUCE that is then passed to TRUNC for actual truncation. A call to FIXMAP returns the desired number NFL of failed tags in the failure vector list.

After truncation, a check (NKP $>0$ ) is made to see if any failure vectors with significant probabilities are left. This may happen, for example, when there are a large number of possible failure candidates all with roughly the same likelihood. If the list is not empty, the new results are transferred to the old arrays for possible use in a subsequent analysis. 


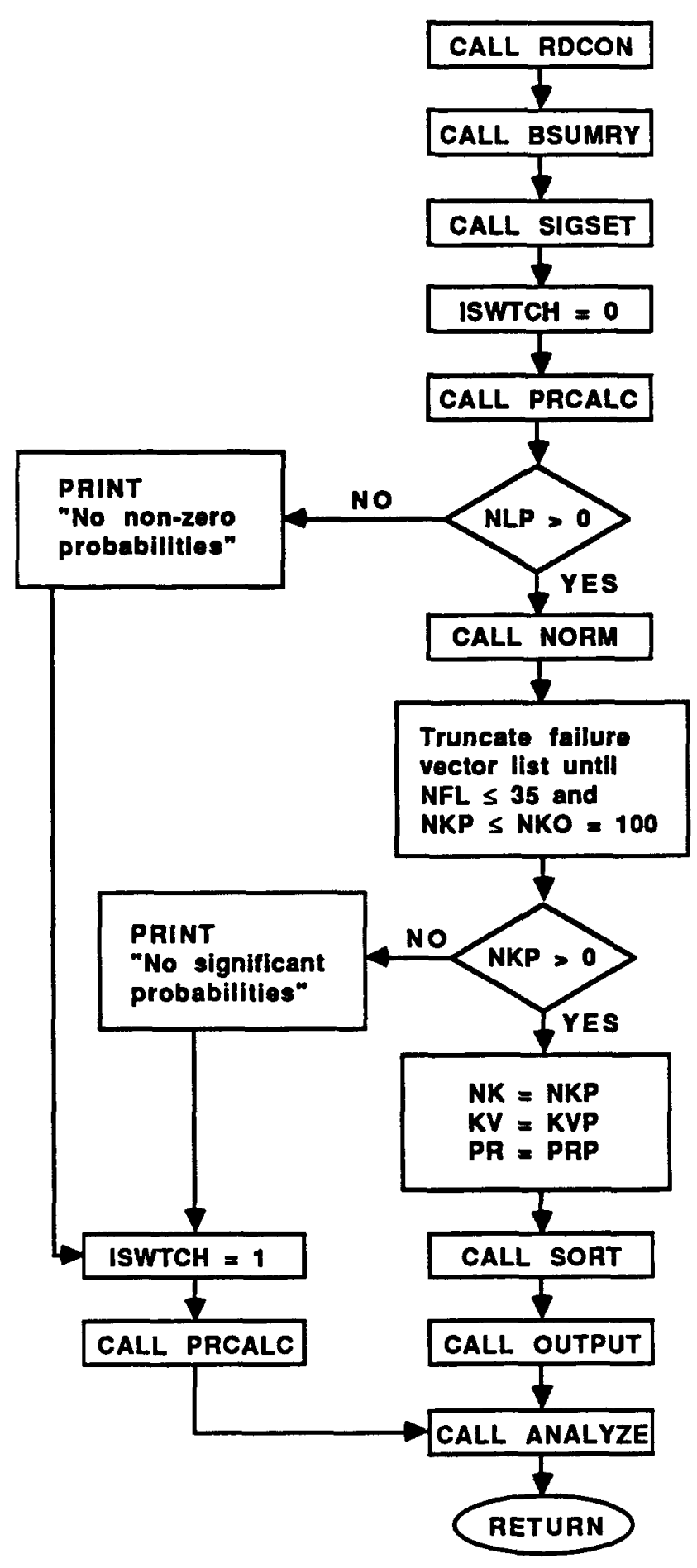

HEDLM $\quad 8702-069.2$

Figure 2. Flow Chart for the Subroutine MEASR. 
The list is then sorted, and the list of fallure vectors is output with the corresponding probabilities. Finally, ANALZE is called to provide a detailed analysis on a case-by-case basis of the most likely candidates for failed tags. In the event of no non-zero or no significant probabilities discussed above, an appropriate message is printed and PRCALC is again called to provide a list of the most likely candidates needed by ANALZE.

The actual subprogram calls are the same for sequential and nonsequential cases. The only difference is that for nonsequential runs the sole initial failure vector with a non-zero probability is the unfailed state. For sequential runs, one starts with $\mathrm{NK}>0$, and all failure vectors $\mathrm{KV}(\mathrm{N})$ represent a system with at least one failed tag. New failure vectors are generated by adding single and double failures to this starting set. The subroutine PRCALC performs this calculation and is thus discussed in Section 3.3 under sequential analysis; however, it is used in nonsequential runs as well.

\subsection{SEQUENTIAL ANALYSIS (PRCALC)}

The subroutine PRCALC generates the desired failure vectors and, based on Equations 1 and 2, calculates the associated probabilities. For computer implementation, these equations may be combined and expressed as

$$
\mathrm{PRP}(\mathrm{KP})=\sum_{\mathrm{K}} \mathrm{PR}(\mathrm{K}) * \mathrm{PIGAM} * \mathrm{PCM}
$$

where the following associations are made.

The arrays $P R P(K P)$ and $P R(K)$ represent $P^{\prime}\left(k^{\prime} / c_{m}\right)$, the final set of probabilities, and $P(k)$, the initial set of probabilities, respectively. The corresponding final and initial failure vectors are stored in the arrays $\mathrm{KVP}(\mathrm{KP})$ and $\mathrm{KV}(\mathrm{K})$, respectively. As noted above, each element of these arrays is actually an array (Dimensioned 4) where each bit represents the failure state of a particular tag. The probability integral $P\left(c_{m} / \tau\right)$ is represented by PCM.

The variable PIGAM combines the transition and channel probabilities

$$
\text { PIGAM }=T\left(k \rightarrow k^{\prime}\right) P\left(\tau / k \rightarrow k^{\prime}\right)
$$

In order to reduce and organize the calculation, the following procedure is adopted. For each initial failure vector $K V(K)$, final failure vectors $K V P(K P)$ are generated by the addition of all possible single and double tag failures or leaks. The number of failed tags represented by a 
failure vector $\mathrm{KV}$ is limited only by the total number of tags; it is only the transition from one state to another that is restricted. In this context, PIGAM may be expressed as

$$
\operatorname{PIGAM}= \begin{cases}\operatorname{PIGAM}(\mathrm{I}) & \text { (single tag leak/failure) } \\ \operatorname{PIGAM}(\mathrm{I}) * \operatorname{PIGAM}(\mathrm{J}) & \text { (double tag leak/failure) }\end{cases}
$$

where PIGAM(I) represents SPI(I) (the a priori failure probability for tag $I$ ); or if tag $I$ is already failed in the failure vector $K V$ under consideration, PIGAM(I) represents GAM(I), the a priori leak probability. This expression for PIGAM also assumes that the values SPI(I) and GAM(I) are small compared with one.

Equation 19 has a simple interpretation. The probability PRP for the final state to exist is the probability that the initial state existed times the probability that the transition to the final stated occurred summed over all initial states. The probability PIGAM * PCM that the transition occurred is the a priori probability that it would have occurred times the probability of obtaining the observed concentrations $c_{m}$ given that it did occur.

In principle, the calculation implied by Equation 19 can now be performed by forming a double DO-loop with the tag indices $I, J$ to cycle through all possible single/double-leak or tag failure transitions. (Single transitions may be denoted by $\mathrm{I}=\mathrm{J}$.) Because of the sum in Equation 19, this approach is impractical. The same final vector KVP will be generated at different times requiring a costly search or indexing scheme. Instead the tag index range was partitioned into two parts, a lower group that includes all tags that are failed in a least one of the failure vectors $\mathrm{KV}(\mathrm{K})$, and an upper group that includes the tags that are unfailed in all failure vectors.

To arrange the tags into failed and unfailed groups, a mapping IW = LMAP(I) is defined that maps the tag index I into a new tag index IW such that the failed tags are all contained in the range IW $=1$ to NFL where NFL is the number of tags that are failed in at least one of the NK initial failure vectors $\mathrm{KV}(\mathrm{K})$. A subroutine FIXMAP calculates NFL and generates the mapping array LMAP. The double DO-loop over tag indices I,J can now be partitioned into four segments with respect to the new indices IW, JW as shown in Table 3 . 
WHC - EP-0190

Table 3. Scheme for Calculating New Failure Vectors KVP(KP).

\begin{tabular}{|c|c|c|c|c|c|}
\hline Segment 1 & Segment 2 & \multicolumn{3}{|c|}{ Segment 3} & Segment 4 \\
\hline $\begin{array}{l}\text { Single } \\
\text { propagation } \\
\text { of prior } \\
\text { failures }\end{array}$ & $\begin{array}{l}\text { Double } \\
\text { propagation } \\
\text { of prior } \\
\text { failures }\end{array}$ & \multicolumn{3}{|c|}{$\begin{array}{l}\text { Single propagation } \\
\text { of prior failures } \\
\text { plus propagation } \\
\text { by new failures }\end{array}$} & $\begin{array}{c}\text { Double/Single } \\
\text { propagation } \\
\text { by new } \\
\text { failures }\end{array}$ \\
\hline$I W-I, N F L$ & $\begin{array}{l}\mathrm{IW}-1, \mathrm{NFL}-1 \\
\mathrm{JW}-\mathrm{IW}+1, \mathrm{NFL}\end{array}$ & $\begin{array}{l}\text { IW-1, NFL } \\
\text { JW-NFL+1 }\end{array}$ & $\begin{array}{l}\text { IW-1, NFL } \\
\text { JW-NFL+2 }\end{array}$ & . . & $\begin{array}{l}\text { IW = NFL+1, NTAG } \\
\text { JW-IW, NTAG }\end{array}$ \\
\hline
\end{tabular}

Segment 1 represents single-failure propagation of existing failure vectors $K V(K)$ for all tags in the failed-tag subspace $I W \leq N F L$. Segment 2 represents double-failure propagation also restricted to the failed-tag subspace. In both cases, a newly created vector KVP may coincide with an old or previously created entry and requires a search by subroutine KVFIND to see. Segment 3 represents double fallure/leak propagation where the first index IW is in the failed subspace and the second index $\mathrm{JW}$ is in the unfailed subspace. This segment consists of blocks that are identical to the first segment with the addition of one new failed tag in the unfailed space (JW > NFL). In Segment 4, both IW and JW are in the unfailed space. In the case that IW = JW, it is possible for a newly generated vector to have been created in Segment 3 and a search is made. Otherwise, the new vector KVP is unique and a search is unnecessary.

\subsubsection{General}

An outline of subroutine PRCALC can now be given and is represented by the schematic flow chart in Figure 3. An initial call to SETSCR sets parameters used later in prescreening to reduce the number of calculations needed. The number NKP of final failure vectors is set to zero, and FIXMAP is called to generate IMAP and NFL. The remainder of the routine is partitioned into the four segments described. If there are no previous failures with non-zero probabilities of occurrence (NFL $=0$ ), as would be the case for a standard nonsequential run, then the beginning list of failure vectors $K V(K)$ is initialized, the number of vectors $N K$ is set to one, $K V(1)$ is set to zero representing no previous failures, and the corresponding probability $\mathrm{PR}(1)$ is set to one. An immediate jump is then made to Segment 4. Otherwise, calculations are performed for the remaining segments.

\subsubsection{Segment 1}

A schematic flow chart for Segment 1 is depicted in Figure 4. The outermost loop is the tag index and ranges over all failed tags. For each 


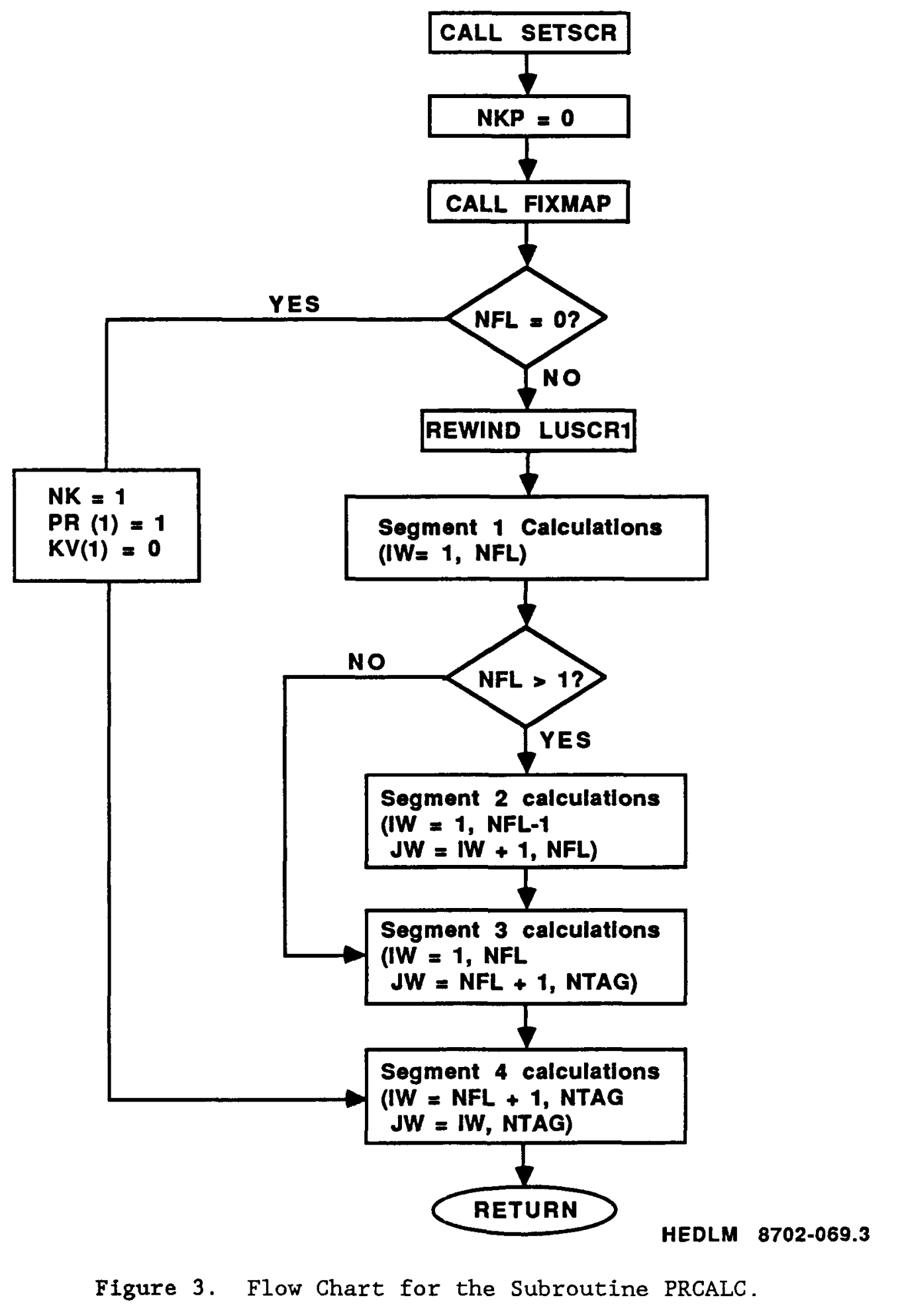




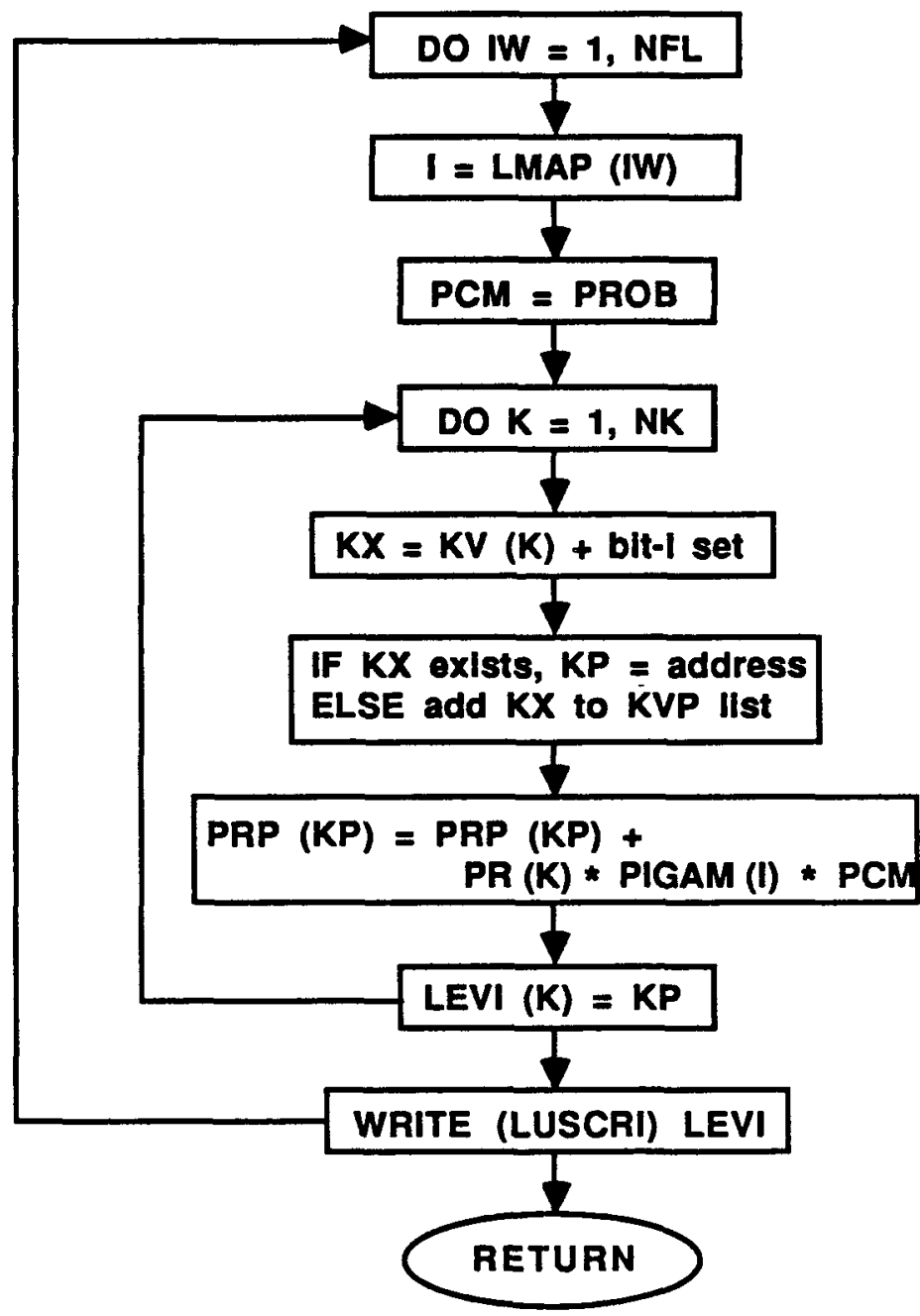

HEDLM 8702-069.4

Figure 4. Flow Chart for the Subroutine PRCALC (Segment 1). 
tag, the function PROB is called to obtain the value PCM of the probability integral $P\left(c_{m} / \tau\right)$. Then for each initial failure vector $K V(K)$, bit $I$ is set to create another failure vector. The new failure-vector list KVP(KP) is searched for the newly created vector, that, if not found, is added to the 1ist. Next, the final probability $P R P(K P)$ is updated and the address of the failure vector is stored in the array LEV1(K) for use in segment 3 . To save direct access memory, LEVI is written to the scratch file LUSCRI = Unit 20.

Not shown in Figure 4 is a call to SETKS that adds tag I to a list of user-specified assured leakers (given in array KS) and creates a combined 1ist NALF of leaking tags used by the function PROB. Another feature not shown is a call to LARGP. This subroutine maintains a list of the NLP largest contributions to the probabilities PRP(KP). This list is later used in MEASR by the ANALZE subroutine to generate part of the final output. For more detail, reference to the code listing is best.

\subsubsection{Segment 2}

This segment (see Figure 5) is very similar to Segment 1, except that the outer DO-loop over failed tags is replaced by a double DO-loop for propagation by double failures or leaks. The same features not explicitly shown described for Segment 1 also apply here, as well as for Segments 3 and 4. In addition, two forms of prescreening are implemented to limit the number of unnecessary calls to PROB, since most of the execution time arises from multiple failure calculations by the PROB subprogram. First, before calling PROB, a call is made to ISCRN to check if the measured concentrations $c_{m}$ are completely inconsistent with the failure mode being considered. Secondly, the probability [one term in Equation 19] that one would find if the probability integral PCM was at its maximum value of unity is tested against a cutoff factor PCUT. If the value is below the cutoff, the term in question is not considered further. For example, the present default values assigned to PCUT and to the a priori failure probabilities SPI(I) are such that simultaneous failure of driver fuel pins is ruled out a priori.

\subsubsection{Segment 3}

Segment 3 (see Figure 6) is closely related to Segment 1 . The outermost DO-loop (JW - NFL+1, NTAG) extends over the range of unfailed tags. For each JW, a block of failure vectors identical to segment 1 , but with bit JW set (failed), is created and added to the KVP(KP) list. By using the address array LEV1 created in Segment 1, the need for repeated searches (as in Segment 1) is eliminated. As before, prescreening and testing against PCUT are done. Segment 3 has the potential to create JW times as many failures vectors as segment 1; therefore, an additional truncation step is introduced within the JW loop to keep the KVP list manageable. A variable IBASE is used to maintain the length of the list. 


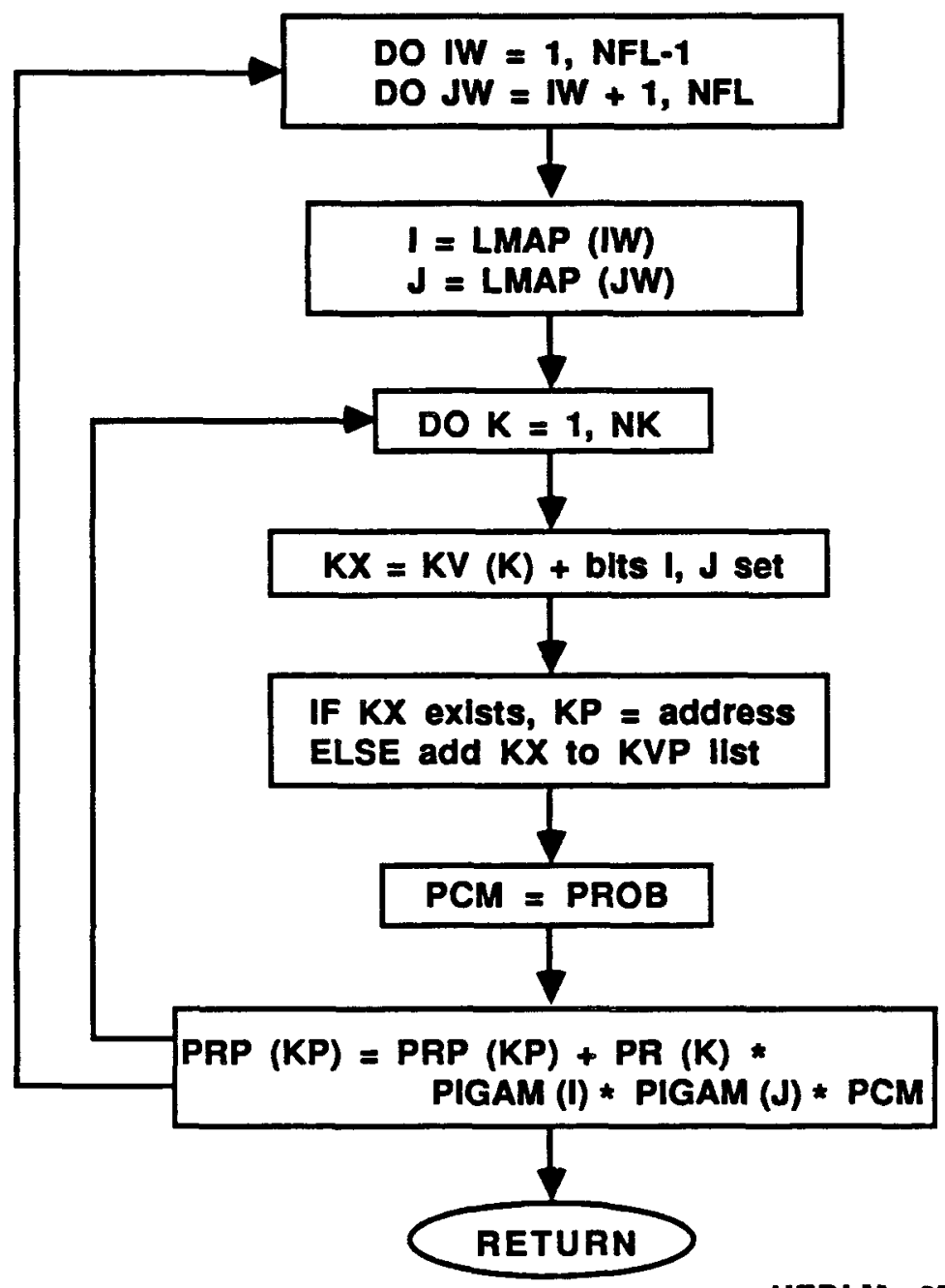

HEDLM 8702-069.5

Figure 5. Flow Chart for the Subroutine PRCALC (Segment 2). 


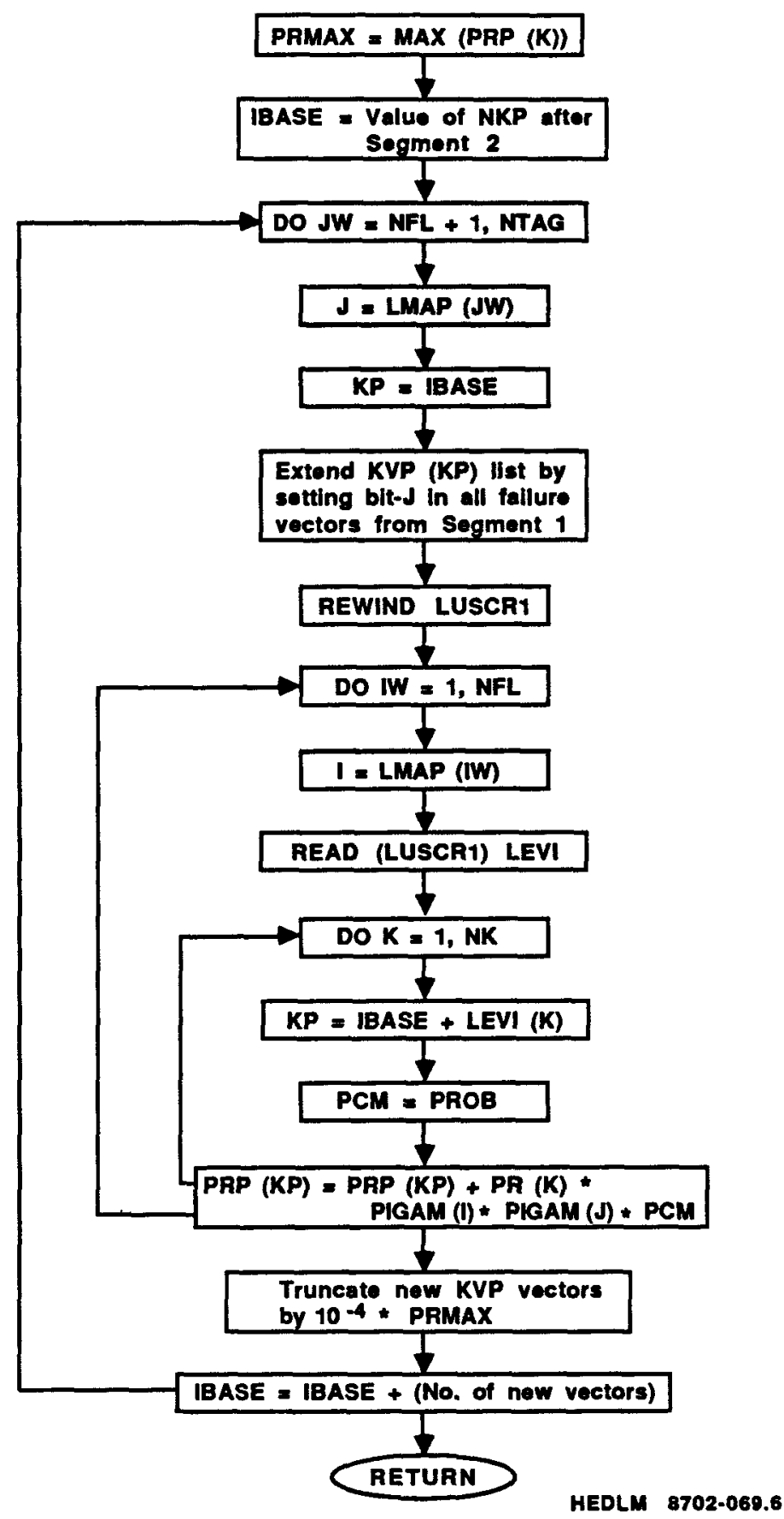

Figure 6. Flow Chart for the Subroutine PRCALC (Segment 3). 
WHC - EP - 0190

\section{3 .5 Segment 4}

The outermost loops in Segment 4 (see Figure 7) are a pair of DO-loops with indices IW and JW that range over the unfailed tags. For double failures, each new failure vector generated is unique, and no searching is needed to see if the new vector was created earlier. Propagation by single failures is included when IW-JW. In this case, it is possible that the newly generated vector was previously created in Segment 3, and a search is made before adding the new vector to the KVP list.

This is the only segment that is always executed and, in particular, is used in a standard nonsequential run where the initial list of failure vectors $K V(K)$ contains no failed tags.

\subsection{PROBABILITY INTEGRAL (PROB)}

The function $P R O B$ is called to evaluate the probability integral $P\left(c_{m} / r\right)$ given in Equation 5 . It may also be called from CPROB if the user selects the PROB keyword to request an analysis for a specific tag failure combination. The calculations are based on the equations following Equation 5 given in Section 2.3, and the code variable names are generally close to the formal names. One exception is the use of $B$ in the code to denote the covariance matrices $M$. A general outline of this routine is shown in Figure 8 . The code listing may be referenced for further details.

The first part of the code assigns a priori values to the leak fractions QO(IGM), and their squared standard deviations SIGQ2(IGM) for al1 leaking components IGM $=1$ to NGM. The first NGM1 are primary components and the remainder are background components. Presently the leak fractions and their standard deviations are both fixed at 0.5 for both tag and fission-product components. These values are specified by the user for background components, but may be defaulted to 1.0 and 0.5 , respectively. The A-matrix, Equation 4, is also initialized with values from the G- and GFP-arrays containing the amounts of tag gas and fission-product gas from the concentration file, respectively.

The next section calculates THC $=\theta_{0}$ (see Equation 14) and completes the calculation of the A-matrix by multiplication by THC for nonbackground components. The covariance matrix for leak fractions is calculated next. It is mainly diagonal with elements given by SIGQ2(IGM); however, within a given tag, fission-product component, or background component, the xenon and krypton subcomponents may be correlated. The correlation parameter may be separately specified for nonbackground and background components and is given by XKRHO and XKRHOB, respectively. Default values are 0.9 and 0.0 respectively, i.e., the amounts (leak-fractions) of xenon and krypton are highly correlated for nonbackground components but uncorrelated for background components. These correlations are less effective than desired for reasons beyond the scope of this discussion. Since the largest submatrix with non-zero off-diagonal elements has dimension two, the inversion of $\mathrm{M}_{\mathrm{q}}$ is carried out explicitly. The final leak-fraction correlation matrix $\mathrm{M}_{Q}$ is then computed, Equation 13, and inverted. 
WHC - EP - 0190

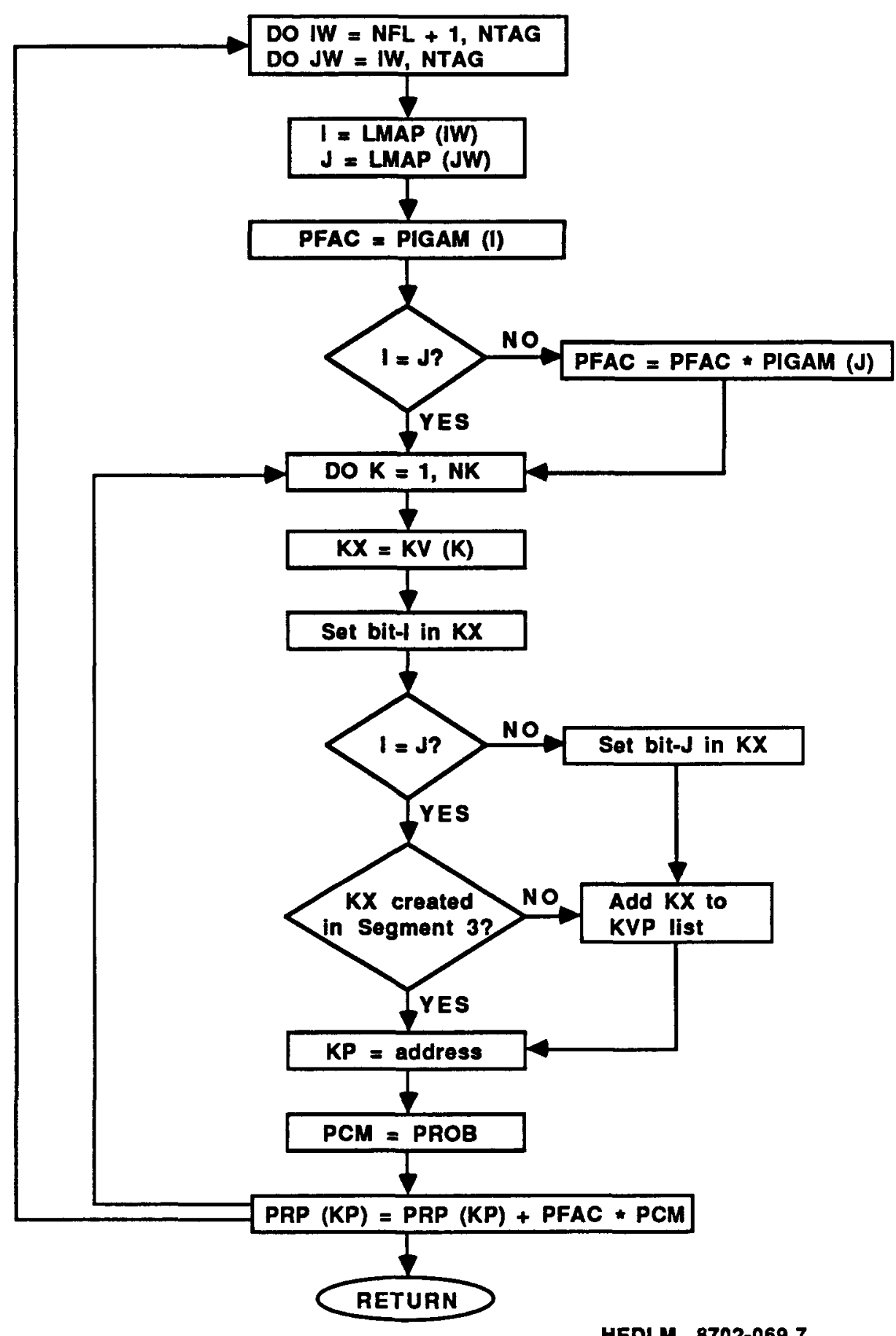

HEDLM $\quad 8702-069.7$

Figure 7. Flow Chart for the Subroutine PRCALC (Segment 4). 


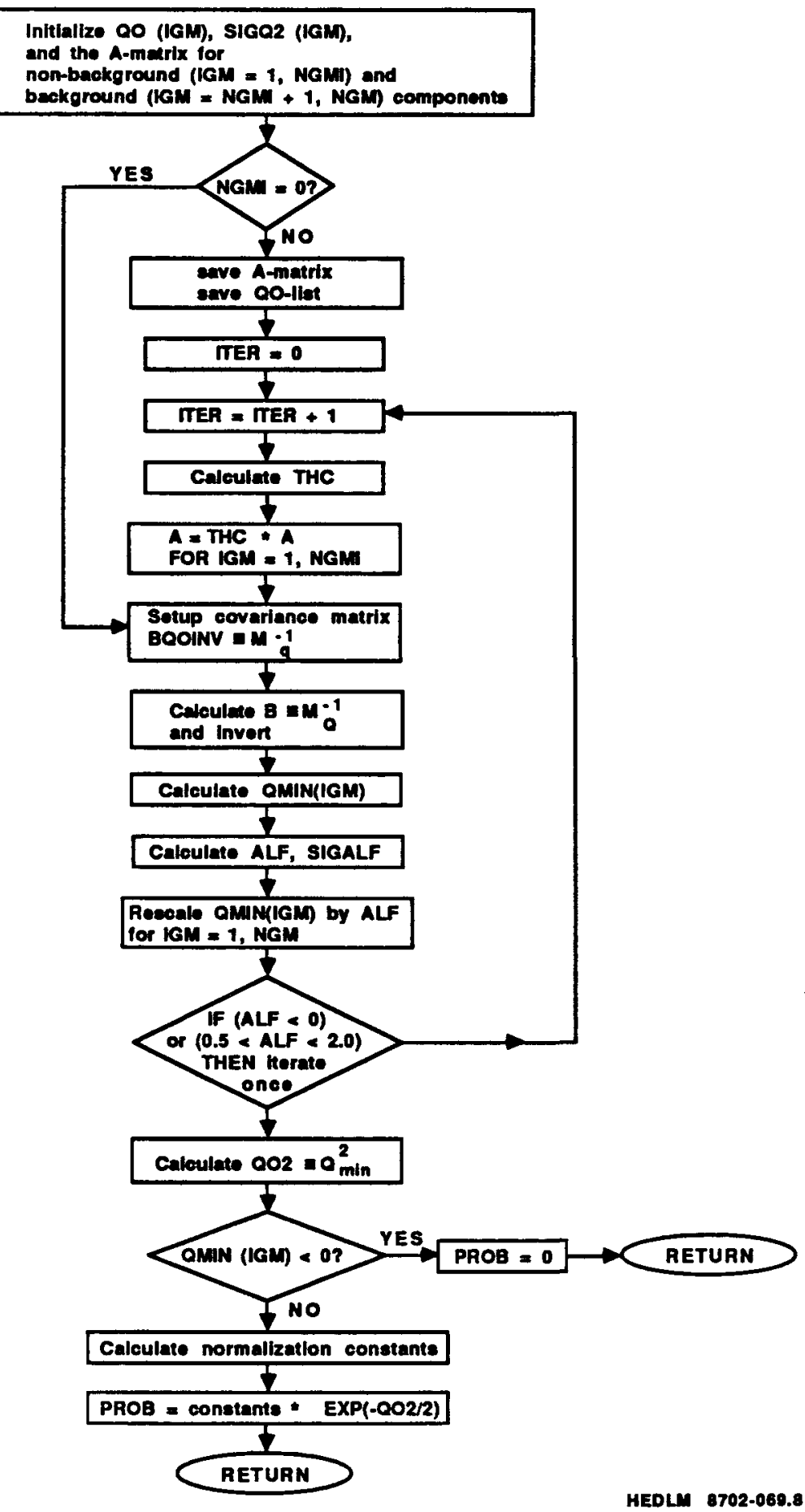

Figure 8. Flow Chart for the Function PROB. 
A least-squares analysis is then completed, Equation 12, to obtain the leak-fractions QMIN(IGM) most consistent with the measured results. These values are later printed out and, as noted earlier, are particularly valuable in assessing the results of the analysis. A correction ALF to be applied to the QMIN values is then obtained along with its uncertainty SIGALF. If this correction differs from unity by more than a factor of two, the whole calculation is iterated one time.

The value $Q 02=Q_{\min }{ }^{2}$, see Equation 11 , is calculated next. It is also output by the code as TOTAL CHI-2. The terms that contribute to Q02 (see Equation 18 and the discussion following that equation) are also output.

Before completing the PROB calculation, a check is made for any QMIN(IGM) < 0 . In this event, PROB is set to zero with an immediate return. This step is not explicitly described in the formalism but is a crucial part of the calculation. For mathematical tractability, Gaussian probability density functions are used to describe the leak-fraction distributions. As a consequence, unphysical negative leak fractions are possible. The rejection of negative QMIN(IGM) strongly mitigates this problem. For simple cases, a comparison of the output from PROB with exact calculations strongly supports this extension to the formalism.

Finally, some miscellaneous normalization constants are obtained, and the calculation is completed. Some logarithms are introduced into the calculation of the constants to solve overflow problems on computers with "real numbers" restricted to be less than $10^{38}$.

A flag ISWTCH that is passed to PROB is not shown. In some cases, a bad measurement for example, Q02 is always so large that the function $\operatorname{EXP}(-Q 02 / 2)$ and hence $\mathrm{PROB}$ is always zero. In order to obtain some useful information, ISWTCH is reset to one (normally, it is zero), and PROB returns the value $\mathrm{Q} 02$.

\subsection{AUXILIARY AND UTILITY ROUTINES}

The focus of this section is on auxiliary routines that are directly related to the operation of the code. The code listing should be referenced for utility routines such as sorting and matrix inversion.

\section{5 .1 LOADG}

This routine reads the concentration file and loads both tag and fission-product inventories into the arrays $G$ and GFP, respectively. The utility routine VLDTYP is also called to select only those tags with the user-specified ITYPEs (also given on the concentration file).

\section{$3.5 .2 \mathrm{RDCON}$}

Measured isotopic concentrations are read for analysis. RDCON is also called by BKGRND to input background concentrations. The total 
WHC-EP-0190

concentration in $\mathrm{p} / \mathrm{b}$ is specified separately for xenon and krypton. Relative amounts for each of the measured isotopes are then entered preceded by a six-character isotope name. They are then rescaled to sum to the total amounts previously given. Thus, the total amounts should include only those isotopes for which relative amounts are specified. Isotopic names given are paired with corresponding names on the concentration file and must match identically. Names not beginning with $\mathrm{KR}$ or $\mathrm{XE}$ are discarded.

\subsubsection{INITL}

Several indices are initialized to zero, and the a priori failure and leak probabilities are set. The a priori failure probabilities for all tags with an ITYPE in the range 20-29 are set to PIOMOT with a default value of 0.001 . The remainder are set to PIODRV with a default value of 0.0001 . With a cutoff probability used in PRCALC of PCUT $=5 \times 10^{-8}$, the possibility of simultaneous double failures of driver tags is ruled out.

\subsubsection{BKGRND}

Up to five background components may be included in an analysis. Each component includes both a krypton and a xenon subcomponent. The BKGRND routine is initiated by the keyword BACKGROUND and provides for adding, deleting, or replacing a component. Values for the leak-fraction $Q B$ and its standard deviation SIGB are first entered followed by an integer specifying the component and operation. Background concentrations are then entered in the format required by RDCON. Isotopes not specified are assigned background concentrations of zero. All values are entered into the CB-array. The isotopic order of this array is governed by the order on the concentration file, and changing concentration files between sequential runs could cause problems. Blank values (see RDREAL) for QB OR SIGB will default to $\mathrm{QBO}-1.0$ and SIGBO $=0.5$, respectively.

\subsubsection{UNCER}

Called by the keyword UNCERTAINTY, this routine resets the relative uncertainties RC(I) that are initialized to RIGCO -0.02 (28) at the beginning of the main module MTAG. The minimum absolute uncertainty SIGCO (see SIGSET) may also be changed from its default value of $0.001 \mathrm{p} / \mathrm{b}$.

\subsubsection{SIGSET}

First the variances (standard deviation squared) SIGC2(I) are obtained from the relative uncertainties $R C(I)$. These values may then be increased for several reasons based on practical experience. If the amount of a given isotope is so small that the absolute uncertainty is less than SIGCO, then the uncertainty is increased to maintain this minimum value.

The isotope ${ }^{80} \mathrm{Kr}$, results of which may be distorted by interference from ${ }^{40} \mathrm{Ar}$ at very small concentrations, is a special case. If the user reduces the floor uncertainty below the default value of $0.001 \mathrm{p} / \mathrm{b}$, then 
the value for ${ }^{80} \mathrm{Kr}$ is set to 0.001 , or five times the new floor value specified by the user, whichever is less. Finally, uncertainties may also be inflated when there is a large spread in the magnitude of the measured concentrations. The absolute uncertainty for a given krypton isotope cannot drop below $5 \times 10^{-6}$ times the total krypton concentration. This factor is $1 \times 10^{-6}$ for xenon.

\subsubsection{CPROB}

As in MEASR, the subroutine CPROB calls RDCON, BSUMRY, and SIGSET to initiate an analysis of measured concentrations from a tag release. Unlike MEASR, which calls PRCALC to analyze and rank all likely candidates for a gas release, CPROB calls PROB directly to provide a detailed analysis of the gas release obtained from any specific tag or combination of tags specified by the user.

\subsubsection{ANALZE/LARGP}

The subprogram PROB is called to give a detailed analysis of the most likely failure combinations. A list of most likely candidates is maintained in the LIP array, which is created by LARGP. An associated array LOCG reorders the list.

\subsubsection{FINDRD}

A reduction factor is found that reduces the number of items in the list PR to a specified range NMIN-NMAX.

\subsubsection{FIXMAP}

A mapping array LMAP, used in PRCALC, is created that reindexes the list of tags. The first NFL tags become all those tags that are failed in at least one of the failure vectors ( $K V(K), K=1, N K)$.

\subsubsection{ISCRN/SETSCR}

Based on selected isotopic ratios, the possible gas concentrations from proposed leaker combinations are compared with the measured concentrations to reduce the number of candidates for which the PROB routine must be called. For each screening ratio, the maximum and minimum possible ratios are found and then extended by three times the assigned uncertainties. If the measured ratios are not in this range, the candidate is rejected (ISCRN $=0$ ). Background components are included in this process and may drastically reduce the effectiveness of screening, because they often allow a large increase in the range of possible (not necessarily probable) values.

The ratios selected for screening are set by SETSCR, which is called near the beginning of PRCALC. 


\subsubsection{VLDTYP/SETTYP}

This pair of routines selects only those tags with a specified ITYPE when they are read from the concentration file in LOADG. VLDTYP is called by LOADG. The specification of ITYPEs is accomplished by SETTYP when the keyword ITYPE is encountered. A feature of SETTYP is that a request for an ITYPE that is a multiple of 10 is expanded over the decade, e.g., an ITYPE request of 20 becomes $20,21, \ldots, 29$. )

\subsubsection{RDREAL/INTRD/RDSTR/RDCRD}

This set of routines provides free format input for all SMTAG entries except the concentration file input. The routines RDREAL, INTRD (an entry point in RDREAL), and RDSTR are for real, integer, and character input, respectively. The function of RDCRD is to input an 80-character buffer named BUFIN, that is acted upon by the other routines.

These routines allow SMTAG entries to occur in arbitrary columns. One difference compared with standard FORTRAN list-directed read input is that entries may exist on multiple input records even when they are part of separate input requests. Another is that character information is entered without enclosing quotes. Thus, embedded blank characters are not allowed for a single entry. Blank characters, commas, and end-of-records all act as data separators. So that a test for blank data can be made where default values are assumed, a pair of commas, separated by blanks at most, returns a length (LEN $=0$ ) to the calling program. 
WHC-EP-0190

\subsection{USAGE}

\subsection{GENERAL}

Detailed instructions for use of the code are given in this section. The input is controlled by the set of keywords listed in Table 1 . The simplest possible nontrivial runstream consists of two keywords:

MEASUREMENT and STOP. The MEASUREMENT keyword initiates the analysis and is followed by the measured isotopic concentrations released to the reactor cover gas.

All input in the runstream is in free format. Both character and numeric values may appear in any column and on as many input records (cards) as desired. Blanks, commas, and the end of the card are all valid data delimiters. Character data are not enclosed in quotes, and therefore must not contain imbedded blanks. In a few cases where default values may be selected by using a blank data field, a pair of commas may be used, separated by, at most, blank characters. Numeric data are also terminated by any character (not including ' $E$ ') not in a valid number.

The isotopic amounts of gas that define each tag reside on the concentration file. It is assumed that all corrections for isotopic burnup, production, and depletion have been made. This file must be assigned to the run as Unit 10 and is a nonbinary text file (see Appendix A for a more detailed description).

For sequential calculations, two other files are used by the code. Unit 11 is used as permanent file to communicate between separate runs. The keyword SAVE causes data to be written to Unit 11, and the keyword SEQUENTIAL reads the data in a subsequent run. Unit 20 is a scratch file used in the subroutine PRCALC.

\subsection{KEYWORDS}

The keywords and associated input data are described in this section. In many cases the order is not fixed, but it should follow a logical sequence. Since the MEASUREMENT keyword initiates an analysis of measured concentrations, it should follow parameter setting options such as resetting the measurement uncertainties. Likewise, the specification of background components should also precede the MEASUREMENT keyword. On the other hand, an UNCERTAINTY keyword could follow one MEASUREMENT keyword to reset the uncertainties for a subsequent MEASUREMENT.

If it is used, the SEQUENTIAL keyword, which causes the results of a previous run to be read from Unit 11, must be the first keyword. The ITYPE keyword, if used, must precede all keywords except the SEQUENTIAL keyword. Keyword STOP, of course, is the final keyword.

As indicated below, many keywords are followed by associated data. Unless otherwise noted, these data may reside on the same input record as the keyword or on following cards as desired. Keywords may be any length, but only the first four characters are checked. In each case below, the 
keyword is the first entry.

In places where the names of isotopes and tags are used, they must match the six-character isotope names and tag idents as defined on the concentration file. The keywords are in boldface type; see below.

\section{SEQUENTIAL}

System parameters (see Section 3.1) are read from Unit 11 where they were saved by a previous SAVE keyword. The result is the same as if the two runs were combined; otherwise, the system parameters are initialized; for example, the number of fallure vectors with failed tags is set to zero. System parameters include Bayesian failure probabilities obtained from the latest MEASUREMENT analysis, background specifications, assured leakers, and a priori failure and leak probabilities. Other data such as reset uncertainties and isotopes labeled as POOR are not saved.

ITYPE $\mathrm{N}$

ITYPE1 ITYPE2 $\ldots$ ITYPEN

Only those tags with the specified ITYPEs are selected from the concentration file for analysis. Any ITYPE that is a multiple of 10 is expanded over the decade. For example, 20 becomes 20-29.

\section{MEASUREMENT DESCRIPTION}

[Measured isotopic concentrations in the RDCON (see below) format.]

The MEASUREMENT keyword is used to enter the isotopic gas concentrations and initiates the analysis. The character variable DESCRIPTION is a user-supplied description of the measurement. Embedded blanks are allowed; this exception to the free format convention requires that DESCRIPTION be the last entry on the card.

\section{BACKGROUND QB SIGB N DESCRIPTION}

[Background gas concentrations in RDCON format.]

The isotopic concentrations of cover gas background components are entered in the RDCON format. Up to five components are allowed. The concentrations of unnamed isotopes are set to zero. The variable DESCRIPTION is a user-supplied description of the background component. As with the MEASUREMENT keyword, embedded blanks are allowed and DESCRIPTION must be the last entry on the card.

$Q B$ is the nominal fractional amount ( $Q B=0$ is acceptable). SIGB is the corresponding standard deviation. If $Q B$ or SIGB are blank (double commas), default values of 1.0 and 0.5 are used, respectively. 
The addition and deletion of background components is controlled by $\mathrm{N}$ :

$$
N= \begin{cases}0 \text { or blank } & \text { add a new component } \\ >0 & \text { replace Nth component } \\ <0 & \text { delete Nth component }\end{cases}
$$

UNCERTAINTY N SIGCO

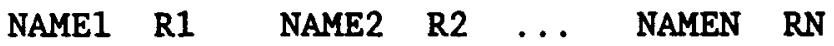

The fractional uncertainties (percent/100) for the named isotopes are reset (default values are $R=0.02$ ). SIGCO is the minimum absolute concentration uncertainty (default is SIGCO- $0.001 \mathrm{p} / \mathrm{b} ;{ }^{82} \mathrm{Kr}$ is a special case).

LEAKER N

NAME1 NAME2 $\ldots$ NAMEN

The $\mathbf{N}$ named tags are treated as assured leakers.

POOR N

NAME1 NAME2 $\ldots$ NAMEN

The named isotopes are not included in the analysis; however, the $\mathrm{C} / \mathrm{Es}$ are computed and printed and contribute to the total $\mathrm{p} / \mathrm{b}$.

CORRELATION XKCORR XKCORRB

XKCORR and XKCORRB specify the correlation between the xenon and krypton leak-fractions for nonbackground and background components, respectively. The default value is 0.9 for nonbackground components and 0 for background components.

SAVE

System parameters are saved (written) to Unit 11 (see SEQUENTIAL).

\section{PRINT TAGDATA}

This pair of keywords causes a short summary of the tag data on the concentration file (Unit 10) to be printed.

PROB

MEASUREMENT DESCRIPTION

[Measured isotopic concentrations in the RDCON format.]

$\mathrm{N}$

NAME1 NAME2 $\ldots$ NAMEN

Repeat until $\mathrm{N}<0$.

Keyword PROB provides an analysis of the specific combination of leaking components defined by the $\mathrm{N}$ tag names NAMEl-NAMEN. The 
calculation is very fast because a single call to the routine PROB is made for the requested leak combination.

The keyword PROB is followed by a standard MEASUREMENT set of cards. The multiplicity $N$ and the desired $N$ leaking tags are then specified. The multiplicity $\mathrm{N}$ and the following tag-ident cards may be repeated as desired until $N<0$. A value $N=0$ is permitted for pure background analysis. The card following $\mathrm{N}^{\prime}<0$ should be a keyword, (e.g., STOP to terminate). All options except LEAKER are valid.

RDCON Format:

NAME-KR NAME-XE P/D-KR P/b-XE N NAME1 CONCEN1 NAME2 CONCEN2 ... NAMEN CONCENN

The first two letters of NAME-KR and NAME-XE should be either KR for krypton or $X E$ for xenon. $P / b-K R$ and $P / b-X E$ are the corresponding total amounts of gas in $\mathrm{p} / \mathrm{b}$ (either may be zero). The names, NAME1-NAMEN, identify the measured relative concentrations CONCEN1-CONCENN. Ratios may be entered by setting one krypton and one xenon concentration to 1.0 .

\subsection{NONSEQUENTIAL EXAMPLE}

\subsubsection{Input}

An example of a nonsequential run is given next. It is terminated by a SAVE command to save the results for input in a sequential run discussed in Section 2.0. The first step is to assign Unit 10 to the concentration file and Unit 11 to a permanent file to save the results. A summary of the input on the standard input unit (card reader) is then given by

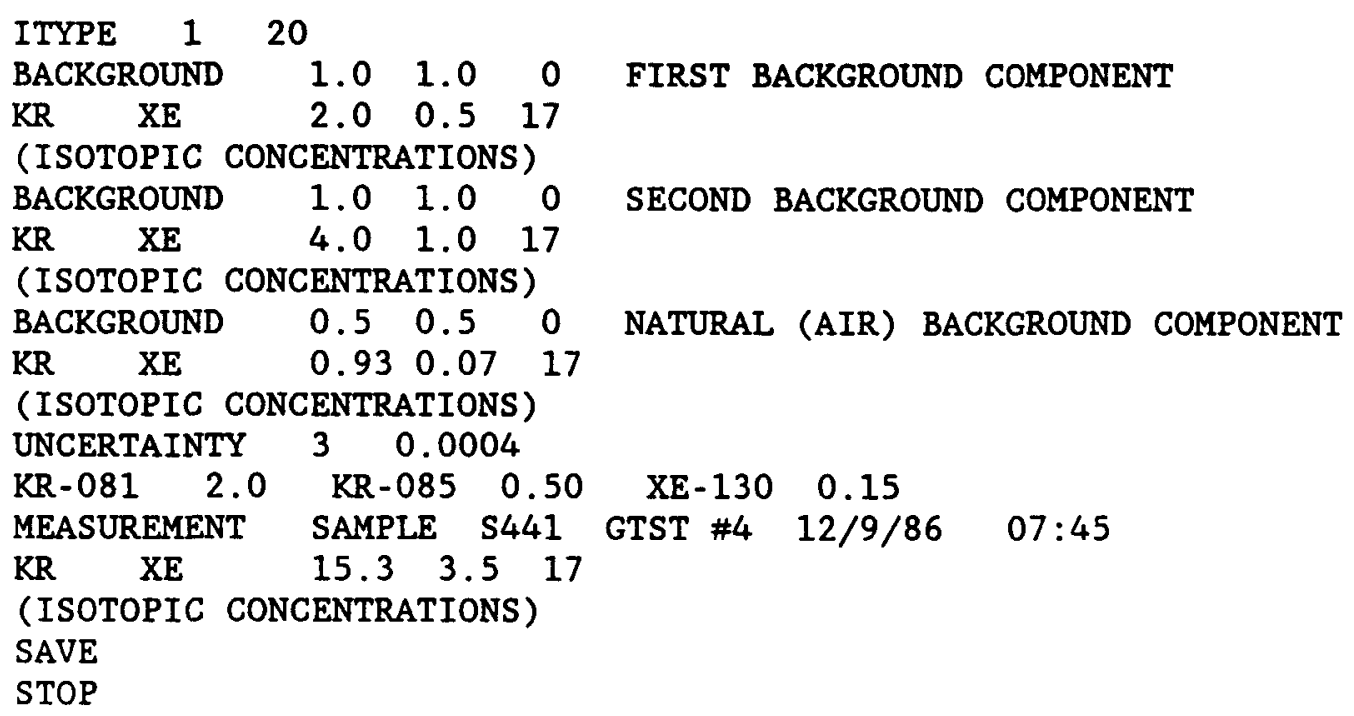

A complete listing with actual isotopic concentrations is given in Appendix B. 
The first input record restricts the analysis to ITYPEs 20-29. This example represents an actual case, and the restriction, based on external radiometric information, improves the execution time. The next three groups of input data are for three separate background components. Uncertainties are then increased for three isotopes. For these tags, the $8 \mathrm{I}_{\mathrm{Kr}}$ concentration was not calculated accurately, and the $200 \mathrm{z}$ (notice not 28) uncertainty effectively eliminates it from the analysis. Perhaps a better option would have been to use the POOR keyword. Notice further that the code does not distinguish between true measurement uncertainties and other sources of error. Thus, all errors that could cause a mismatch between calculated and measured values are lumped into the "measurement" uncertainties. Measured gas concentrations are then entered and the analysis is initiated by a MEASUREMENT keyword. Finally, results are SAVEed for use by a subsequent run, and the run is terminated.

\subsubsection{Output}

The output may be grouped in three sections. A complete output listing for this run is given in Appendix C. The first section is primarily a description of the input and is relatively self-explanatory. Both measured and background isotopic concentrations are given as relative and absolute amounts. Relative values are the input values, and absolute values have been rescaled so that they sum to the total amounts of xenon and krypton specified. The MEASUREMENT input summary also includes a summary of the background components in effect at the time the MEASUREMENT keyword was encountered.

The next part of the output starts with a summary of the number of failure vectors considered by different segments of the PRCALC subroutine. It can be of interest in assessing the operation of the code but in general can be ignored. The desired ranking of possible failures follows.

Failure probabilities are presented in two parts. The first section, labeled as "Bayesian Results," gives the probability for each possible combination of failed tags. The first five entries (see Appendix $C$ for the complete list) in this example are:

\begin{tabular}{|c|c|c|c|c|}
\hline \multicolumn{2}{|c|}{ Bayesian Results } & \multicolumn{3}{|c|}{ Failure Identification } \\
\hline Rank & Probability & TagId & TagId & $\cdots \cdots \cdots+\cdots$ \\
\hline 1 & 0.303 & TAGN23 & & \\
\hline 2 & 0.251 & TAGN24 & & \\
\hline 3 & 0.234 & TAGN32 & & \\
\hline 4 & 0.210 & TAGN22 & & \\
\hline 5 & 0.000 & TAGN24 & TAGN32 & \\
\hline
\end{tabular}

showing roughly equal failure probabilities for four different tags. A double failure is also shown with a negligible probability. 
For this example where the most likely failures are all single failures, global probabilities are nearly identical to the above results:

\begin{tabular}{|c|c|c|}
\hline Rank & Probability & TagId \\
\hline 1 & 0.304 & TAGN23 \\
\hline 2 & 0.251 & TAGN24 \\
\hline 3 & 0.235 & TAGN32 \\
\hline 4 & 0.210 & TAGN22 \\
\hline
\end{tabular}

These probabilities are not disjoint. In other words, the value of 0.304 for TAGN23 is the probability that TAGN23 is failed, independent of whether or not some other tag is also failed.

The third section of the output provides an analysis of the various combinations of leaking components that are most consistent with the measured gas concentrations. It gives a detailed picture of the least-squares solutions given by Equation 12 and calculated in the PROB function. Since at any one step, at most two tags are assumed to leak, unless supplemented by use of the LEAKER keyword, each analysis is identified by the one or two tags assumed to leak. This is in contrast to the above probability table where each entry represents the tags in the reactor that are failed at that point in time. The two descriptions are the same only if there were no previous failures at the beginning of the analysis.

This section is further divided into two parts: a leak-fraction analysis and a concentration analysis (see Appendix $C$ ). In the first part, the leak-fractions $\left\{q_{m i n}, \alpha\right\}$ as determined by the least-squares solution, Equation 12, are given along with their standard deviations for each leaking component [see Equation 4]. The leak-fractions are usually near their a priori values for valid runs and should always be carefully examined. An exception is when two tags leak very different amounts so that their leak-fractions must differ. A leak fraction for a background component that differs substantially from the value given a priori by the user is cause to re-examine the background assumptions. A value that is outside the assumed uncertainties will also cause a larger value in the CHI-2 column; see the discussion after Equation 18.

The first leak analysis given in Appendix $C$ is for TAGN23 by itself. Including both the tag and three background components as well as the xenon and krypton subcomponents, there are a total of ten leaking components that combine to form the final gas mixture; up to 20 are allowed. This particular tag does not belong to a fuel pin and does not have fission products, although they are included in the analysis (their value is set to zero on the concentration file). Note that the fission-product concentration (in the column PPB) is zero and also that the final standard deviation given in column five is equal to the priori 
value of 0.5 . The lack of a reduction in the standard deviation shows that the measured concentrations do not fix the size of the fission-product leak fractions.

For background components, the reduction or lack thereof in the leak fraction standard deviation is an excellent diagnostic to show whether or not a particular component is needed by the analysis. For example, the krypton component for the second background is much better fixed than the xenon component. Both krypton and xenon leak fractions for this background are also larger than the a priori value of 1.0 , showing that the size of this background component was slightly underestimated. This conclusion is valid, however, only if the leaking tag was in fact TAGN23.

The concentration analysis follows and provides an isotope-by-isotope comparison between the results of the least-squares analysis and the measured values. The agreement of the calculated and experimental values is shown by the $\mathrm{C} / \mathrm{E}$ column. Good agreement is also shown by relatively small values in the CHI- 2 column. The uncertainty column shows the input standard deviations in percent used in the analysis. In the analysis for TAGN23, ${ }^{82} \mathrm{Kr}$ has the default value of 28 , while the uncertainty for $130 \mathrm{Xe}$ has the value 158 that was reset with the UNCERTAINTY keyword. For other isotopes such as ${ }^{78} \mathrm{Kr}$, the code has increased the uncertainty. Note in the latter case that the 22.88 uncertainty corresponds to an absolute uncertainty of $0.0004 \mathrm{p} / \mathrm{b}$ as specified by SIGCO, a value also reset from its default value by the UNCERTAINTY keyword.

The final part of the analysis includes the total CHI-2 and its separate contributions from the leak-fraction and concentration analysis $\left[Q_{\min }{ }^{2}\right.$ in Equation 11]. This value is then used in Equation 10 to obtain a final unnormalized probability. Values for ALPHA and its standard deviation are also output. The value for ALPHA is usually near one, and a much different value $(e . g ., 0.5)$ is cause to question the results.

\subsection{SEQUENTIAL EXAMPLE}

The measured gas release in the previous example narrowed the likely failure candidates to four. A larger gas release occurred a day and a half later. Results of this second measurement are represented by the following input cards:

\section{SEQUENTIAL}

\begin{tabular}{lllllll} 
ITYPE 1 & \multicolumn{1}{l}{20} & & & & \\
UNCERTAINTY & 3 & 0.0004 & & & \\
KR-081 2.0 & KR-085 & 0.50 & XE-130 & 0.15 & \\
MEASUREMENT & SAMPLE & S442 & GTST \#2 & $12 / 10 / 86$ & $21: 34$ \\
KR XE & 130.0 & 23.5 & 17 & & & \\
(ISOTOPIC CONCENTRATIONS) & & & & \\
STOP &
\end{tabular}

See Appendix B for the complete input listing. The keyword SEQUENTIAL causes the system parameters, including the failure probabilities from the previous analysis, to be read from Unit 11. The ITYPE and UNCERTAINTY keywords and data are repeated. Note that the background concentrations, 
which are considered part of the system description, are not specified; however, they are passed along from the first run. The same results could have been obtained by including the second measurement in the first runstream.

Results from the second analysis are:

\begin{tabular}{ccccc}
\multicolumn{2}{c}{ Bayesian Results } & & \multicolumn{2}{c}{ Failure Identification } \\
\cline { 4 - 5 } Rank & Probability & TagId & TagId & $\ldots \ldots \ldots \ldots$. \\
1 & 0.751 & TAGN32 & & \\
2 & 0.099 & TAGN23 & TAGN32 & \\
3 & 0.082 & TAGN24 & TAGN32 & \\
4 & 0.068 & TAGN22 & TAGN32 & \\
\hline
\end{tabular}

These results indicate that the most likely scenario is that only TAGN32 is failed. In other words, TAGN32 failed for the first measurement and leaked again for the second measurement. Nevertheless, there is a nontrivial probability that one of the other tags (TAGN22, TAGN23, or TAGN24) is also failed along with TAGN32. The occurrence of the pairs of failure candidates does not imply that they failed together, only that by the time of the second measurement, there was a small probability that each member of indicated pair had failed at some time.

TAGN32 shows up in every entry, and therefore, its probability for failure is 100\%. This shows up also in the example of global probabilities:

\begin{tabular}{ccc} 
Global & Probabilities & \\
Rank & Probability & TagId \\
\hline 1 & 1.000 & TAGN32 \\
2 & 0.099 & TAGN23 \\
3 & 0.082 & TAGN24 \\
4 & 0.068 & TAGN22 \\
\hline
\end{tabular}

A complete output listing is given in Appendix $C$.

\subsection{PROB EXAMPLE}

From the above analyses it appears that TAG32 was the dominant contributor to the second measurement. Suppose that one wanted to check the possibility that TAGN22 also contributed. Since a detailed leak analysis for the TAGN22-TAGN32 combination was not output, a PROB analysis would provide the desired output. The input runstream would be as follows:

BACKGROUND $\quad 1.0 \quad 1.0 \quad 0 \quad$ FIRST BACKGROUND COMPONENT 
WHC - EP - 0190

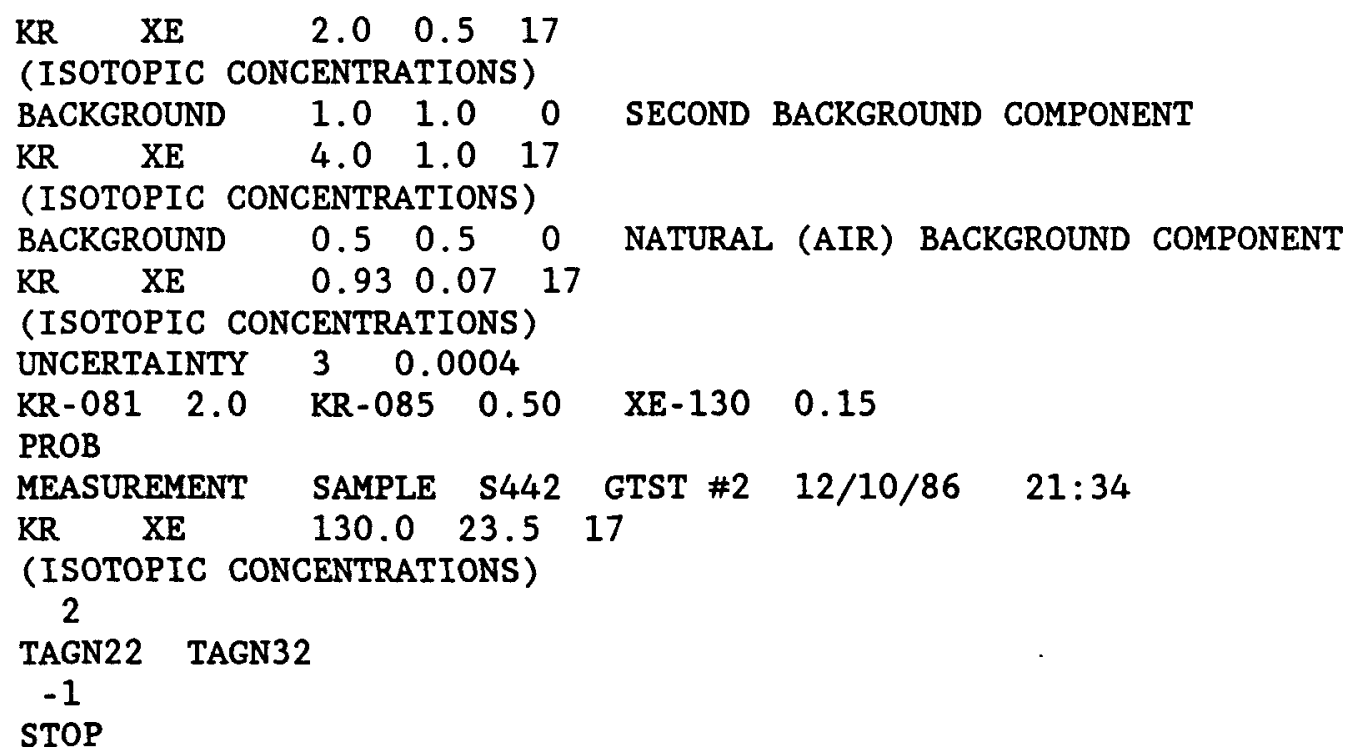

First, the backgrounds are entered as before. Since only the specified PROB analysis is performed, there is no incentive to use the ITYPE selection. The keyword PROB and the MEASUREMENT cards are then given, followed by the desired number $N$ of leaking tags and the tag identifiers. Additional analyses can be requested until $\mathrm{N}$ is negative. A background analysis that includes only background components can be obtained with $\mathrm{N}=0$, a convenient option when no component has recently leaked gas. Another advantage of the PROB option is that the number $N$ of multiple leakers may exceed two (as long as the total number of leaking components does not exceed 20), so that higher order simultaneous failures can be analyzed if needed.

Results of this analysis (not shown) give a very large value for CHI-2. Moreover, some of the background leak fractions have negative values that are physically impossible. Thus, one concludes that the measured concentrations are completely incompatible with the assumption that tags TAGN22 and TAGN32 leaked together.

\subsection{NOTES}

Some guidelines for the interpretation of the results were provided in the previous examples and experience has provided a few other items. One difficulty that can arise is the use of background components that are as large or larger than the measured concentrations. The conversion factor that relates the cover-gas concentration to the amount of gas that leaks from the fuel pin (or other component) is estimated by first subtracting the a priori estimates of the background gas already in the cover gas from the measured values [see Equation 14]. Consequently, negative and unphysical estimates for $\theta_{0}$ may occur if the background is large and overestimated. 
Because of a bad measurement or other possible input error, one may get a message indicating that no non-zero or no significant probabilities were found (see the flow chart in Figure 2). Some supplementary analysis should follow that can help spot inconsistences in the input data. In any event, it is necessary to correct the input before drawing any conclusions.

In a system with high trapping efficiency for both xenon and krypton, the ratio of krypton to xenon in the gas sample can be reliable enough to provide some discrimination in the identification of different tags. The xenon to krypton amounts correlation in SMTAG gives a higher probability to tags with ratios near the measured values but is not as effective as could be desired. Hence, an examination of the leak fractions for xenon and krypton can, in some cases, be used to reject otherwise possible failure candidates. The relative leak fractions for tag and fission-product isotopes can also give a good indication of the fission-product release-to-birth factor for a fuel pin failure. These data may be useful in ascertaining the character of the fuel pin breach as well as the fuel pin burnup.

Some final comments on the use of nontag isotopes are in order. In principle, the tag system depends on only a few selected tag isotopes; in practice, there are many benefits to tracking all available stable isotopes. For example, age-tagging discrimination based on burnup is automatically taken into account. Also, in the event of contamination by air (which contains tag isotopes), the inclusion of nontag isotopes allows the least-squares method to pinpoint the amount of air in the sample and to automatically account for it in the analysis. For unfueled reactor components, it has been found useful to use fission-product isotopes as tags, thus requiring their inclusion in the analysis.

When many isotopes are included, additional redundancy is gained. On the other hand, the analysis is also more sensitive to indirect effects, and greater care in specifying background components may be required. For example, when the analysis is restricted to the primary tag isotopes, a gas sample contaminated with a very small amount of air might well yield the correct tag identification, even if air were not included as a background component. On the other hand, with an expanded set of isotopes, the failure to include air as a background component could easily cause the code to give an indeterminate result because of inconsistencies in the secondary isotopes. 


\subsection{REFERENCES}

1. Figg, J. A., and R. Sanchez, 1980, Reactor Assembly Failure Location Analysis Program (FLAP) User's Guide, HEDL-TME 82-36, Hanford Engineering Laboratory, Richland, Washington.

2. Figg, J. A., N. J. McCormick, R. E. Schenter, and F. Schmittroth, 1980, "Gas Tag Identification of Failed Reactor Assemblies - IV Analysis Methods," Nuclear Technology, 50:18.

3. Gross, K. C., 1980, Polyfail: A Program for Identification of Multiple Failures with Gas Tagging, ANL-80-87, Argonne National Laboratory, Oakbrook, Illinois.

4. Lambert, J. D. B., B. Y. C. So, F. S. Kern, J. R. Armstrong, E. R. Ebersole, and M. T. Laug, 1978, "Recent Improvements in Identifying Fission Product Sources in the Experimental Breeder Reactor II, Nuclear Technology, 39:275.

5. Schmittroth, F., 1973, "A Probabilistic Method for Identifying Failed Fuel Elements Using a Gas Tag System," Nuclear Science and Engineering, $52: 95$.

6. Schmittroth, F., 1986, "Location Analysis for Multiple Gas Tag Releases," Nuclear Science and Engineering, 93:88-96. 
WHC-EP-0190

A P P E N D I X A

CONCENTRATION FILE

A-1 


\section{APPENDIX A}

\section{CONCENTRATION FILE}

The concentration file contains the isotopic amounts that define the tag system. The format is not free and must follow the specifications given here. Since only the relative gas concentrations are important, the amounts may be in any convenient units such as number of atoms or $\mathrm{cm}^{3}$ of gas.

As shown below, the beginning of the file contains a title card, the number of isotopes on the file, and the isotope names. These six character names are used to match names specified in the SMTAG free format input and should therefore not contain imbedded blanks.

The number of reactor assemblies is then given, followed by the tag data for each assembly. Each assembly includes a header record followed by the data for 1 to KTAG distinct tags. The data for each tag consist of two sections: the isotopic amounts in the tags, and the isotopic amounts generated as fission products. All names isotopes are in both sections. The assembly header card includes, in order: a six character identifier, the ITYPE (all tags within an assembly must have the same ITYPE), the number of tags in the assembly, and two other variables not presently used.

TITLE

NI

(INAME (I), I $-1, N I)$

N55MB

DO $N=1, N 55 \mathrm{MB}$

ASEM, MTYP, KTAG, FLNC, IDAA

DO $\mathrm{K}=1, \mathrm{KTAG}$

TAGID, (G(I), I=1,NI)

(GFP(I), I=1,NI)
(20A4)

(10x, I5)

$(9 \mathrm{X}, 8(1 \mathrm{X}, \mathrm{A6}))$

(10X, I5)

$(4 \mathrm{X}, \mathrm{A} 6,10 \mathrm{X} 2 \mathrm{I} 4,15 \mathrm{X}, \mathrm{E} 13.5, \mathrm{I} 5)$

$(4 \mathrm{X}, \mathrm{A} 6,5 \mathrm{X}, 5 \mathrm{E} 13.6 /(15 \mathrm{X}, 5 \mathrm{E} 13.5))$

$(15 \mathrm{X}, 5 \mathrm{E} 13.6)$

END $\mathrm{K}-L O O P$

END N-LOOP 
WHC-EP-0190

\section{APPENDIX A}

PARTIAL LISTING OF THE CONCENTRATION FILES

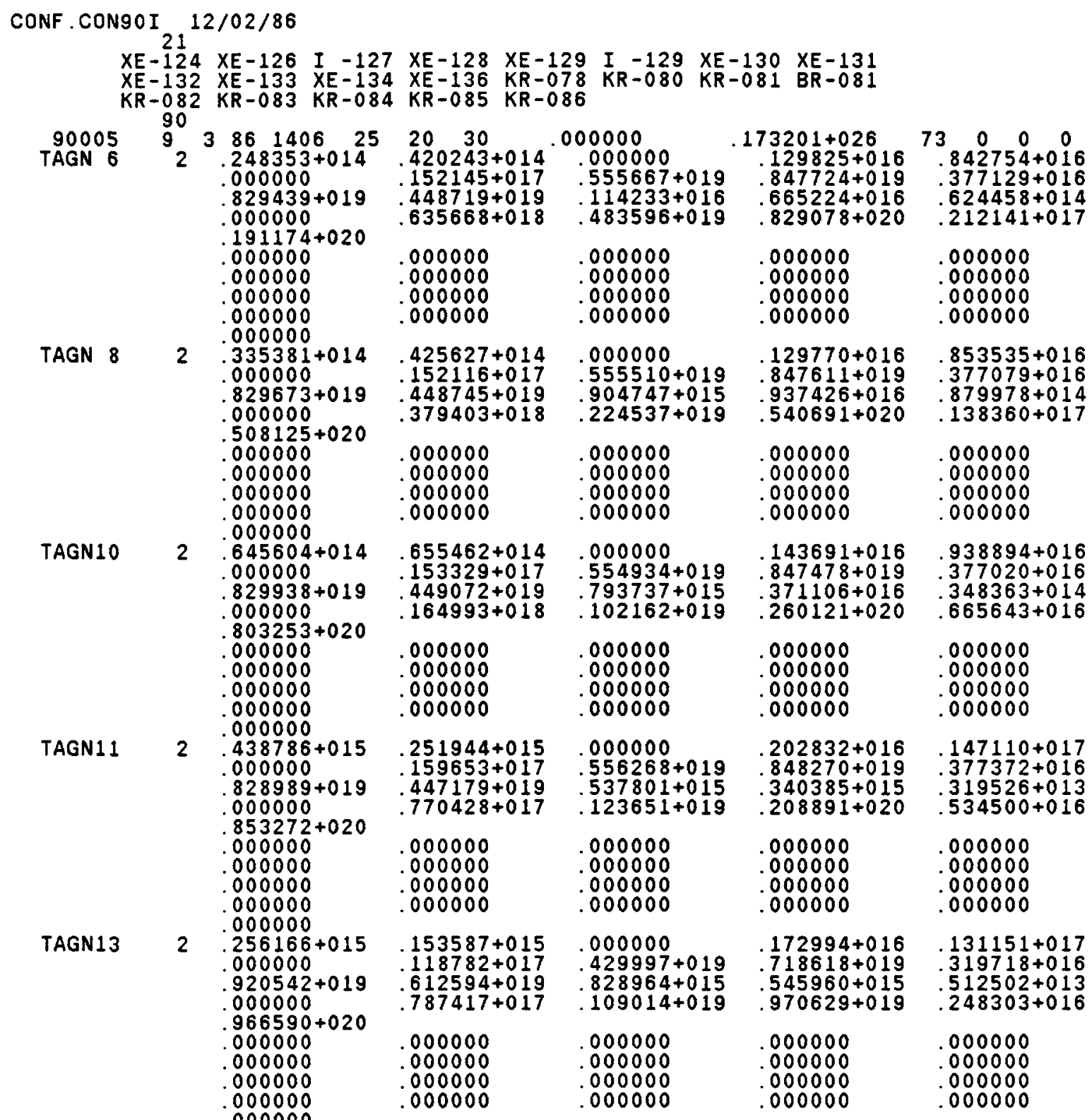


WHC-EP-0190

A P P E N D I $X \quad B$

EXAMPLE INPUT DATA

B-1 


\section{APPENDIX B}

NON-SEQUENTIAL INPUT

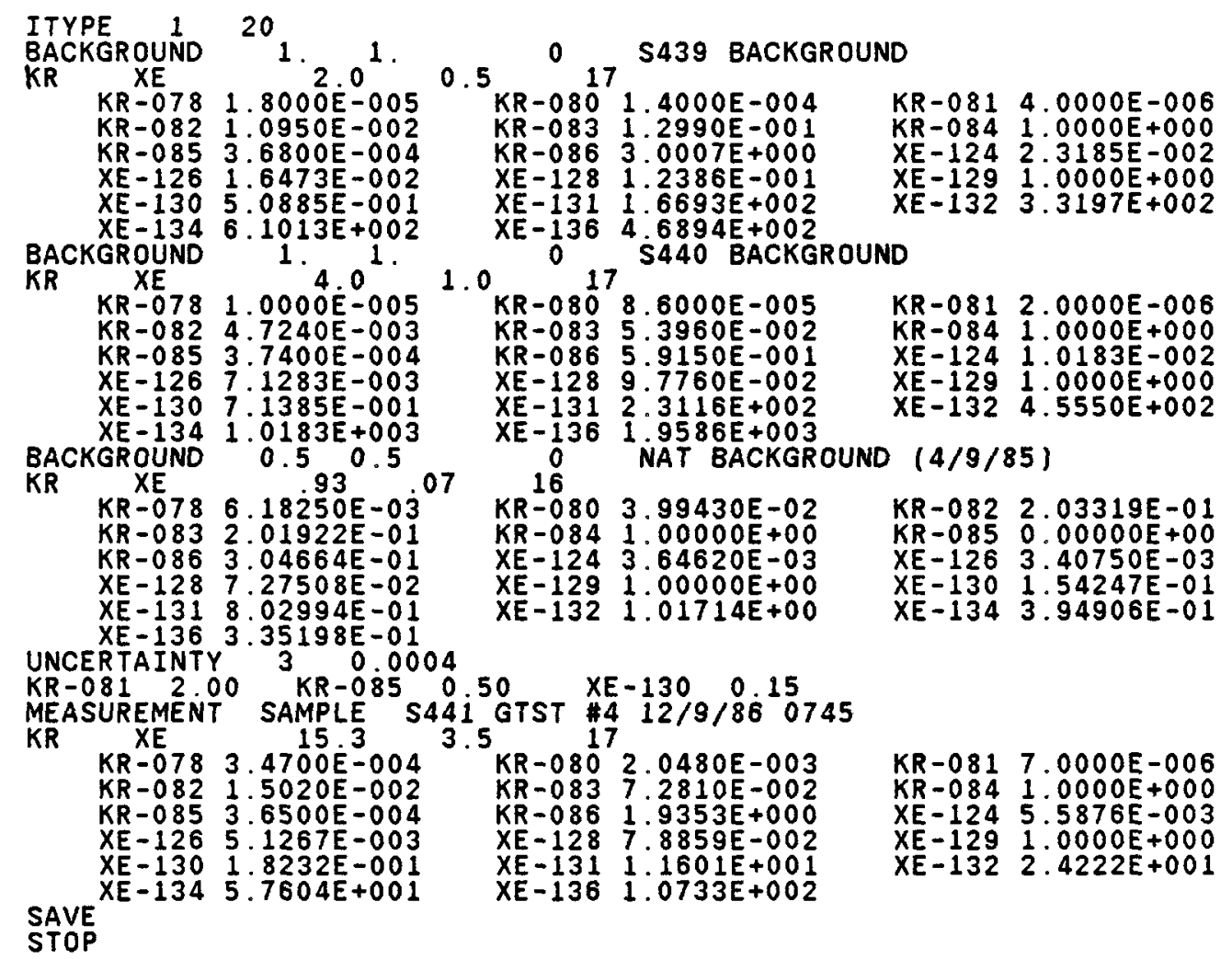

SEQUENTIAI INPUT

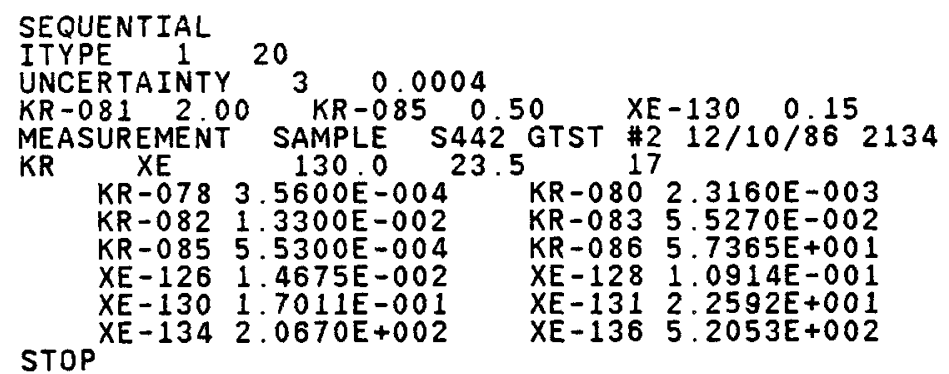

KR-0813.4000E-005 $K R-084 \quad 1.0000 E+000$ $X E-1242.0670 E-002$ $X E-12910000 E+000$ $X E-1325.8661 E+001$ 
WHC-EP-0190

$\begin{array}{llllllllll}A & P & P & E & N & D & I & X & C\end{array}$

EXAMPLE OUTPUT DATA 


\section{APPENDIX C}

NON-SEQUENTIAL OUTPUT

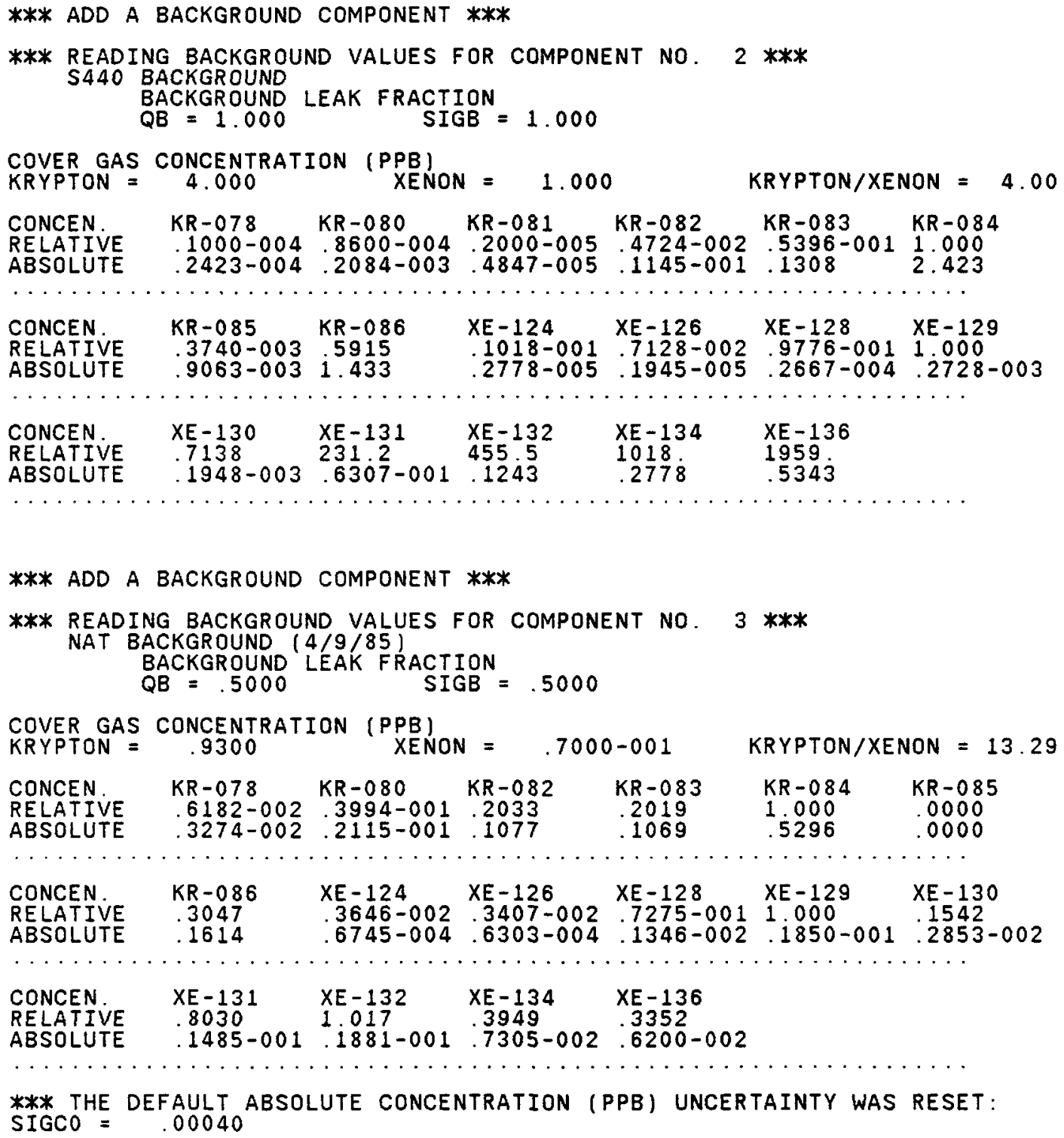




\section{APPENDIX C}

EXAMPLE OUTPUT

QXQT

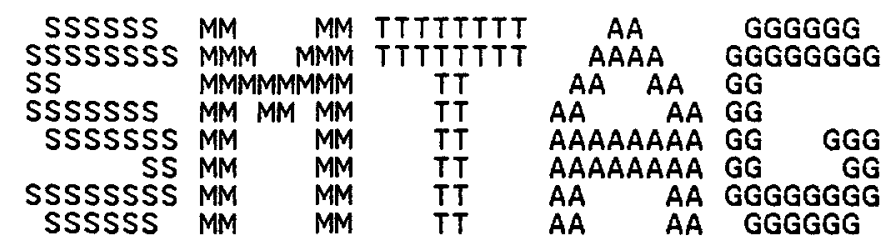

VERSION 241 DECEMBER 12,1986

$\begin{array}{llrlrlllll}* * * & \text { THE } & \text { FOLLOWING ITYP'S } & \text { ARE } & \text { SELECTED } & * * * * & & & \\ 20 & 21 & 22 & 23 & 24 & 25 & 26 & 27 & 28 & 29\end{array}$

*** READING CONCENTRATION FILE ****

CONF CON9OI $12 / 02 / 86$

NUMBER OF TAGS ON CONCENTRATION FILE $=0$

NUMBER OF TAGS SELECTED BY ITYPE $=30$

*** INITIALIZING SYSTEM PARAMETERS ***

*** ADD A BACKGROUND COMPONENT ***

*** READING BACKGROUND VALUES FOR COMPONENT NO 1 **** S439 BACKGROUND $\mathrm{BACKGROUND}$
$\mathrm{QB}=1000$

COVER GAS CONCENTRATION (PPB)

\begin{tabular}{|c|c|c|c|c|c|c|}
\hline $\begin{array}{l}\text { COVER GAS } \\
\text { KRYPTON }=\end{array}$ & $\begin{array}{l}\text { CONCENTRAT: } \\
2000\end{array}$ & $\begin{array}{r}\text { ION (PPB) } \\
\text { XENON }\end{array}$ & 500 & & KRYPTON/XEI & NON $=$ \\
\hline $\begin{array}{l}\text { CONCEN } \\
\text { RELATIVE } \\
\text { ABSOLUTE }\end{array}$ & $\begin{array}{l}K R-078 \\
1800-004 \\
8691-005\end{array}$ & $\begin{array}{l}\mathrm{KR}-080 \\
1400-003 \\
6760-004\end{array}$ & $\begin{array}{l}K R-081 \\
4000-005 \\
1931-005\end{array}$ & $\begin{array}{l}K R-082 \\
1095-001 \\
5287-002\end{array}$ & $\begin{array}{l}\mathrm{KR}-083 \\
1299 \\
6272-001\end{array}$ & $\begin{array}{c}K R-084 \\
1000 \\
4828\end{array}$ \\
\hline $\begin{array}{l}\text { CONCEN } \\
\text { RELATIVE } \\
\text { ABSOLUTE }\end{array}$ & $\begin{array}{l}K R-085 \\
3680-003 \\
1777-003\end{array}$ & $\begin{array}{l}K R-086 \\
3001 \\
1449\end{array}$ & $\begin{array}{l}X E-124 \\
2319-001 \\
7339-005\end{array}$ & $\begin{array}{l}X E-126 \\
1647-001 \\
5214-005\end{array}$ & $\begin{array}{l}X E-128 \\
1239 \\
3921-004\end{array}$ & $\begin{array}{l}X E-129 \\
1000 \\
3165-003\end{array}$ \\
\hline $\begin{array}{l}\text { CONCEN } \\
\text { RE LAT IVE } \\
\text { ABSOLUTE }\end{array}$ & $\begin{array}{l}X E-130 \\
5089 \\
1611-003\end{array}$ & $\begin{array}{l}X E-131 \\
1669 \\
5284-001\end{array}$ & $\begin{array}{c}X E-132 \\
3320 \\
1051\end{array}$ & $\begin{array}{l}X E-134 \\
6101 \\
1931\end{array}$ & $\begin{array}{c}X E-136 \\
4689 \\
1484\end{array}$ & \\
\hline
\end{tabular}


WHC-EP-0190 *** THE FOLLOWING
KR -081
2 ORACTIONAL CONCENTRATION UNCERTAINTIES WERE RESET

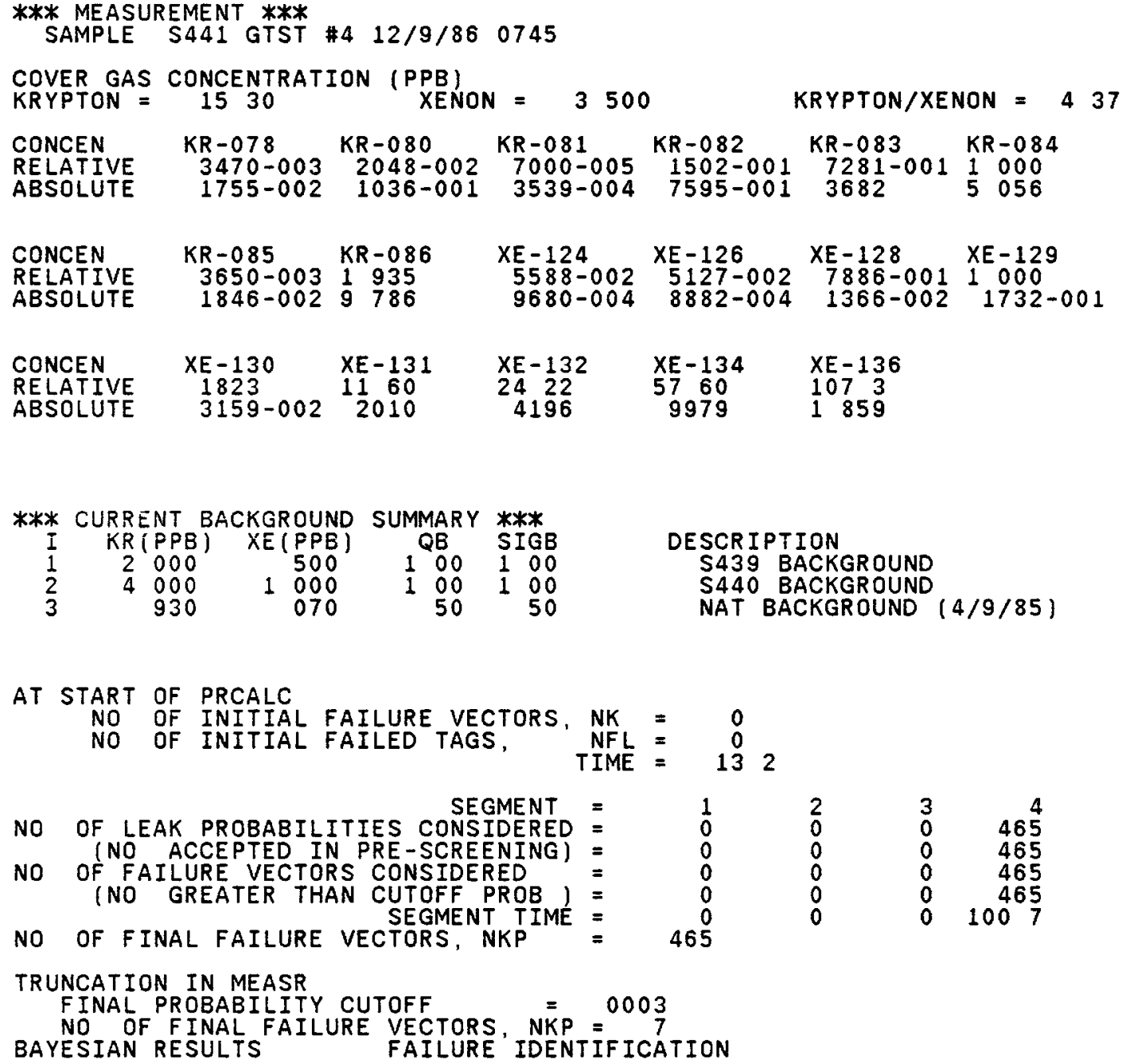


WHC-EP-0190

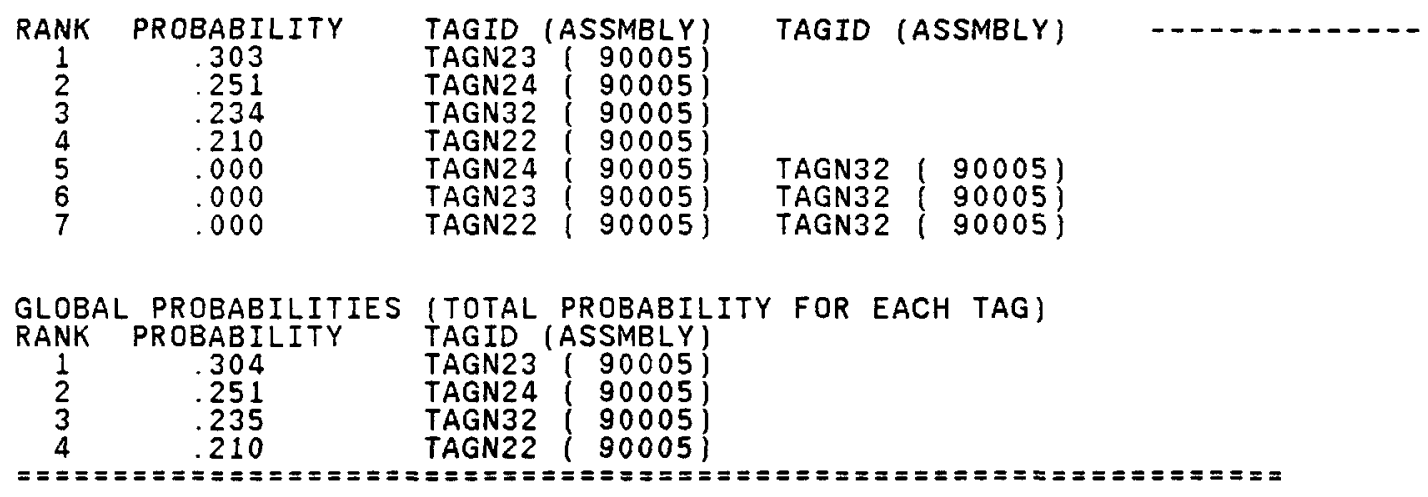

*** DETAILED LEAK ANALYSIS ****

FAILURE IDENT $=$ TAGN23 $(90005)$

UNNORMALIZED PROBABILITY $(K / C M)=.1814-010$

* leak fraction analysis * THETA, (PPB/ATOMS) =

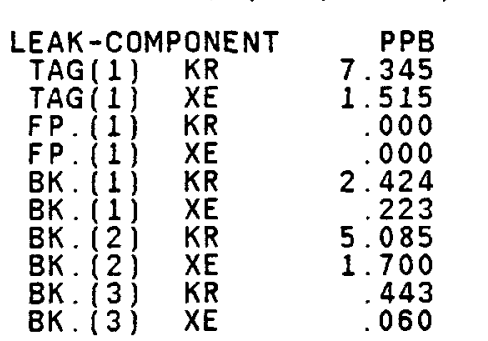

$\begin{array}{ccc}1502 & \\ \text { LEAK-FRACTION } & \\ \text { VALUE } & \text { STD.DEV. } & \text { CHI -2 } \\ .455 & .029 & .04 \\ .375 & .057 & .33 \\ .511 & .500 & .00 \\ .511 & .500 & .00 \\ 1.212 & .269 & .04 \\ .446 & .211 & .31 \\ 1.271 & .066 & .07 \\ 1.700 & .319 & .49 \\ .477 & .016 & .00 \\ .862 & .022 & .52\end{array}$

* CONCENTRATION anAlySis *

* CONCENTRATION

\begin{tabular}{|c|c|c|c|c|}
\hline $\begin{array}{l}\text { ISOTOPE } \\
K R-078 \\
K R-080 \\
K R-081 \\
K R-082 \\
K R-083 \\
K R-084 \\
K R-085 \\
K R-086 \\
X E-124 \\
X E-126 \\
X E-128 \\
X E-129 \\
X E-130 \\
X E-131\end{array}$ & $\begin{array}{l}\text { MEASURED } \\
1755-002 \\
1036-001 \\
.3539-004 \\
.7595-001 \\
3682 \\
5.056 \\
1846-002 \\
9.786 \\
.9680-004 \\
.8882-004 \\
.1366-002 \\
1732-001 \\
.3159-002 \\
.2010\end{array}$ & $\begin{array}{c}\text { CALC /MEAS } \\
\text { i. } 189 \\
1.017 \\
.2659 \\
.9998 \\
\text { i9999 } \\
1.000 \\
.8989 \\
.9996 \\
.9335 \\
.8355 \\
.9700 \\
1.001 \\
.9599 \\
.9949\end{array}$ & $\begin{array}{c}\text { UNCERT }(\%) \\
22.80 \\
9.66 \\
1130.12 \\
2.00 \\
2.00 \\
2.00 \\
50.00 \\
2.00 \\
413.22 \\
450.37 \\
29.28 \\
2.31 \\
15.00 \\
2.00\end{array}$ & $\begin{array}{r}\mathrm{CHI}-2 \\
.13 \\
.03 \\
.00 \\
.00 \\
.00 \\
.00\end{array}$ \\
\hline
\end{tabular}


WHC-EP-0190

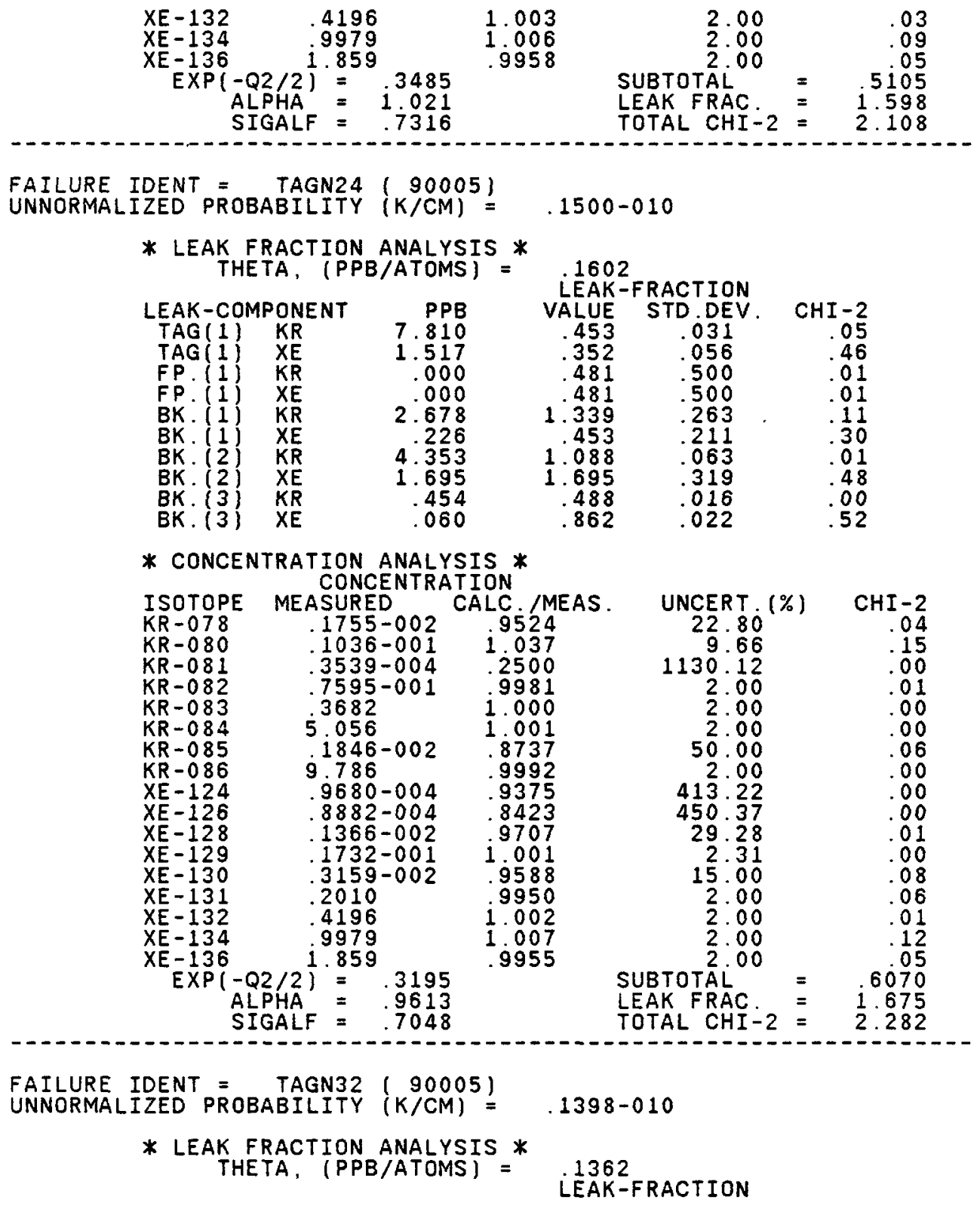


WHC-EP-0190

$\begin{array}{lrrrrr}\text { LEAK-COMPONENT } & \text { PPB } & \text { VALUE } & \text { STD.DEV } & \text { CHI-2 } \\ \text { TAG (1) } & \text { KR } & 5.025 & .343 & .021 & .52 \\ \text { TAG }(1) & \text { XE } & 1.530 & .418 & .061 & .14 \\ \text { FP. (1) } & \text { KR } & .000 & .535 & .500 & .03 \\ \text { FP. (1) } & \text { XE } & .000 & .535 & .500 & .03 \\ \text { BK. (1) } & \text { KR } & 3.296 & 1.648 & .247 & .42 \\ \text { BK. (1) } & \text { XE } & .694 & 1.388 & .334 & .15 \\ \text { BK. (2) } & \text { KR } & 6.570 & 1.642 & .078 & .41 \\ \text { BK. (2) } & \text { XE } & 1.217 & 1.217 & .395 & .05 \\ \text { BK. (3) } & \text { KR } & .419 & .450 & .015 & .01 \\ \text { BK.(3) } & \text { XE } & .058 & .834 & .023 & .45\end{array}$

* concentration analysis *

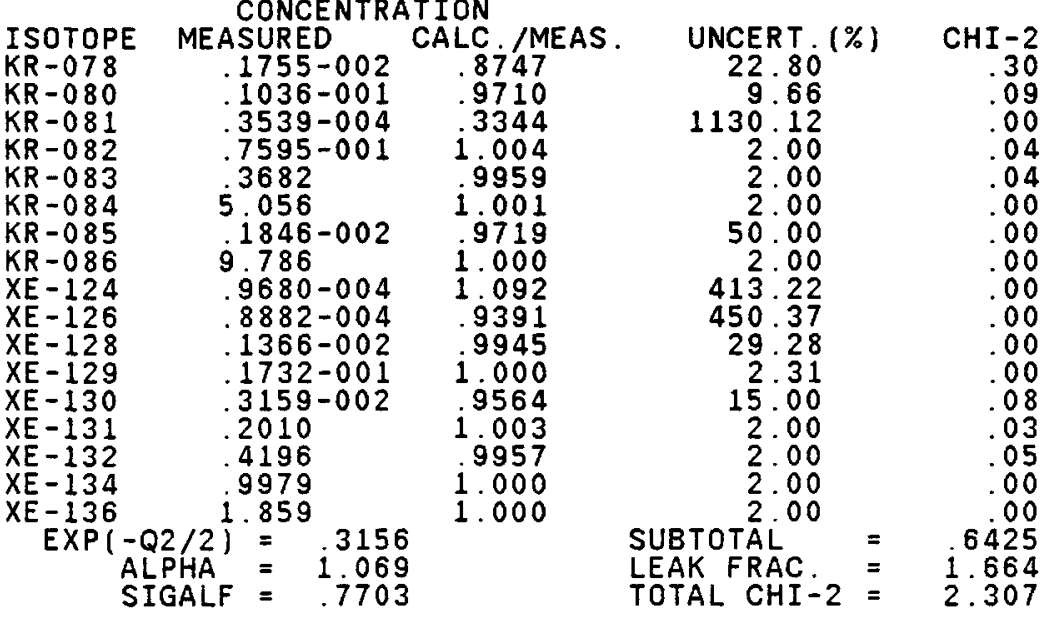

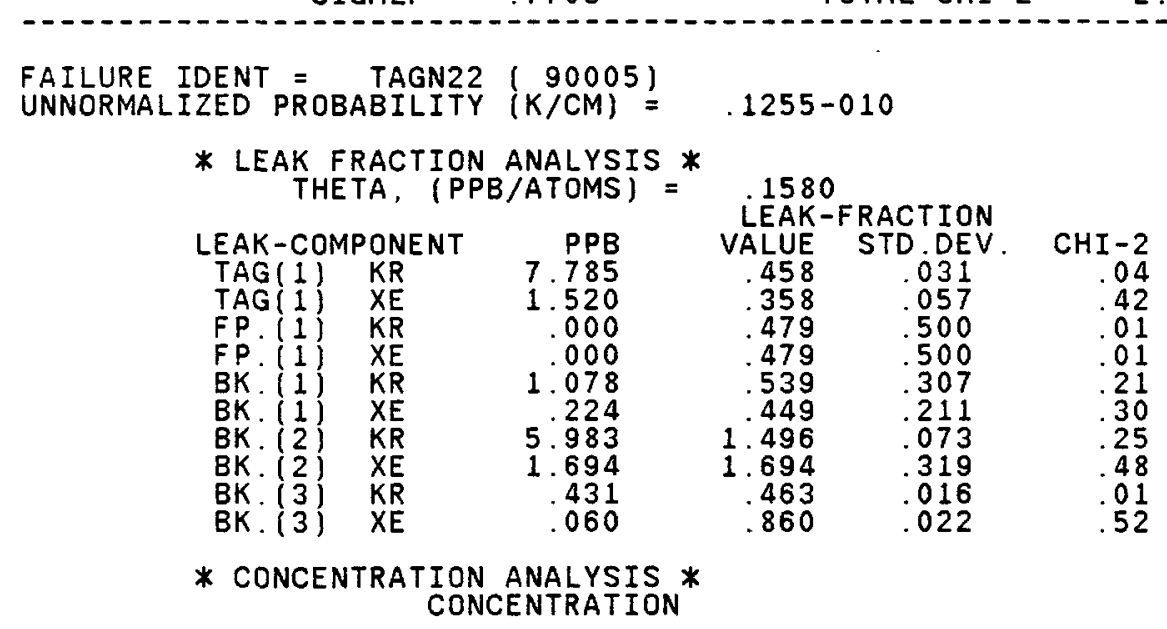


WHC-EP-0190

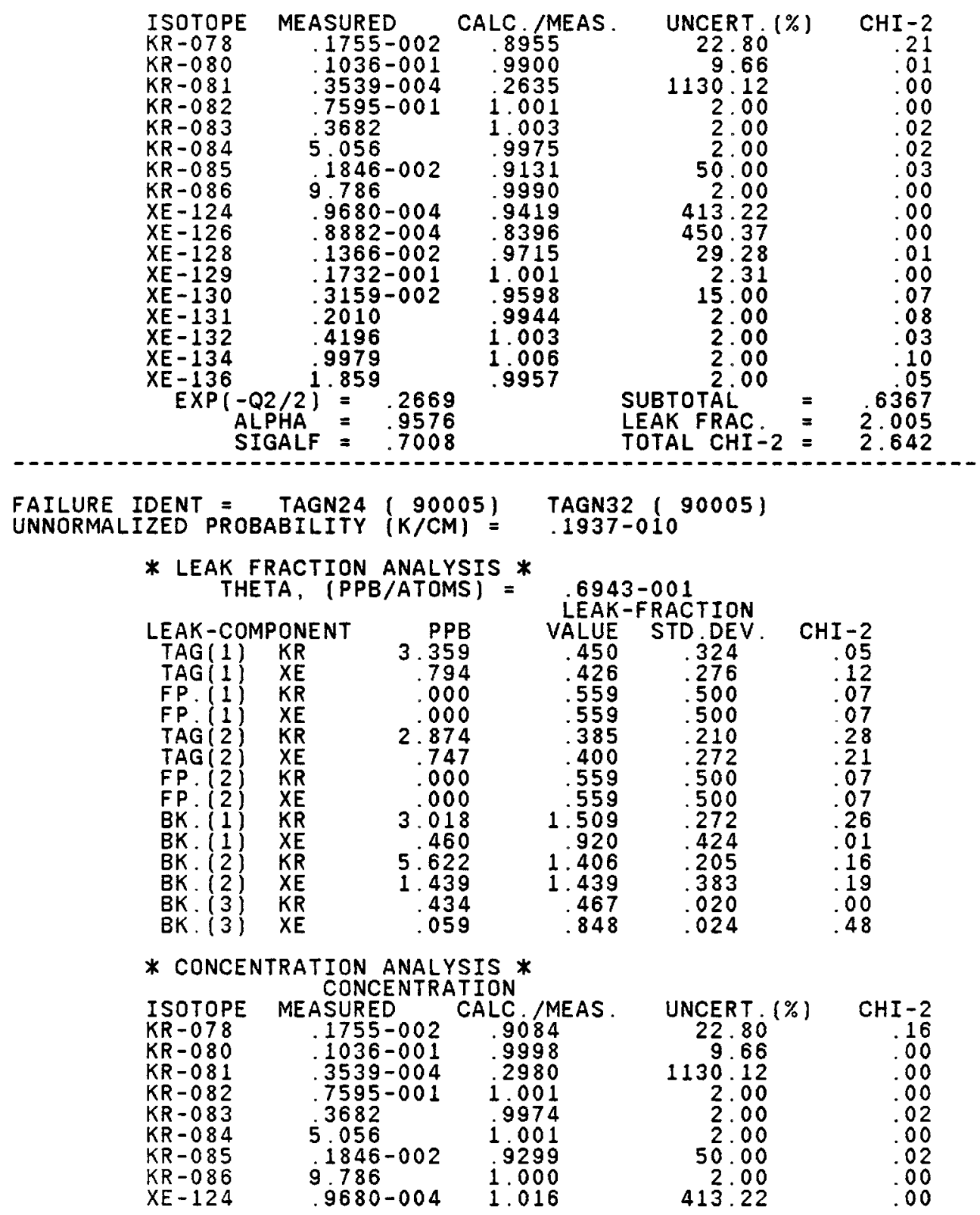


WHC-EP-0190

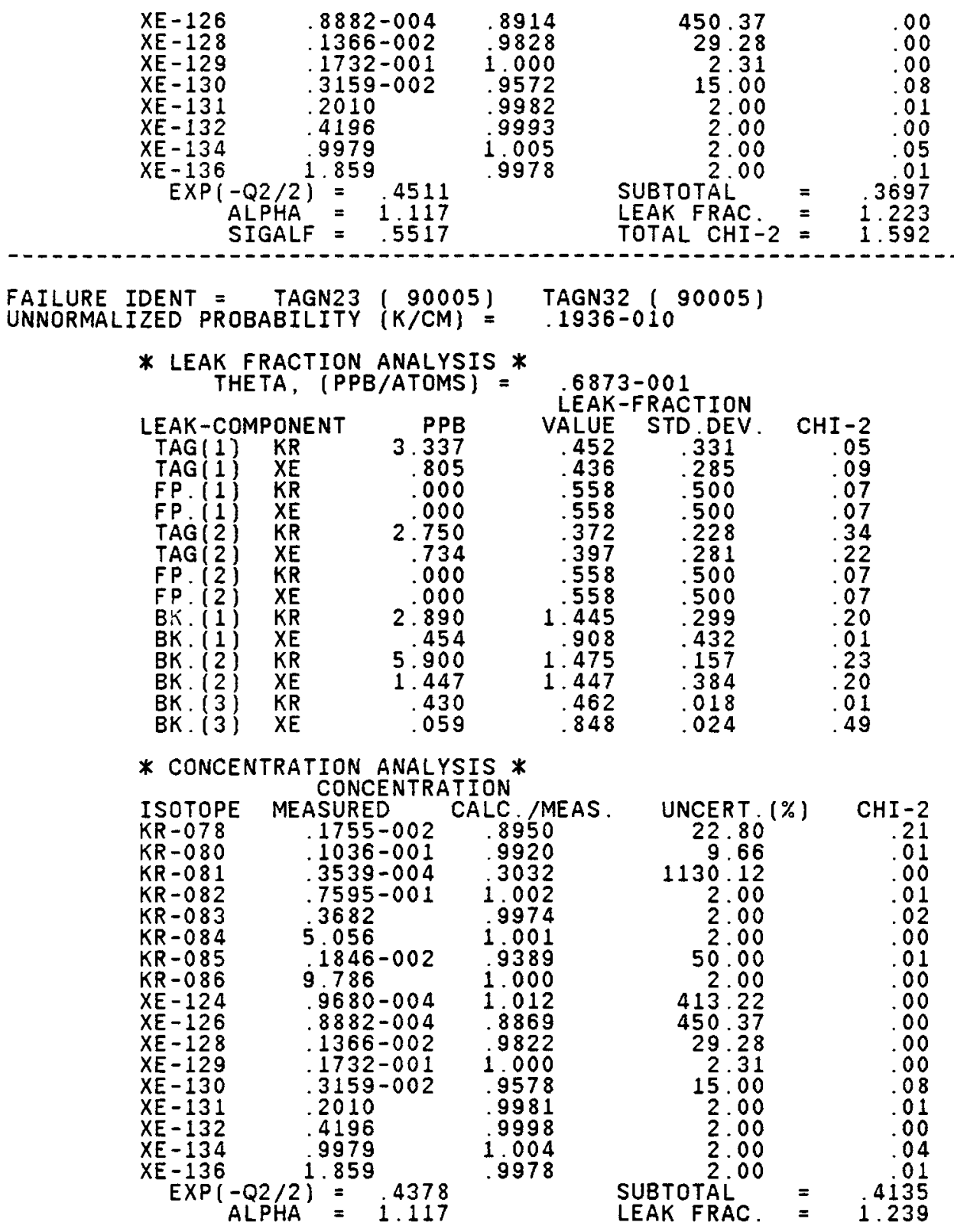


WHC - EP - 0190

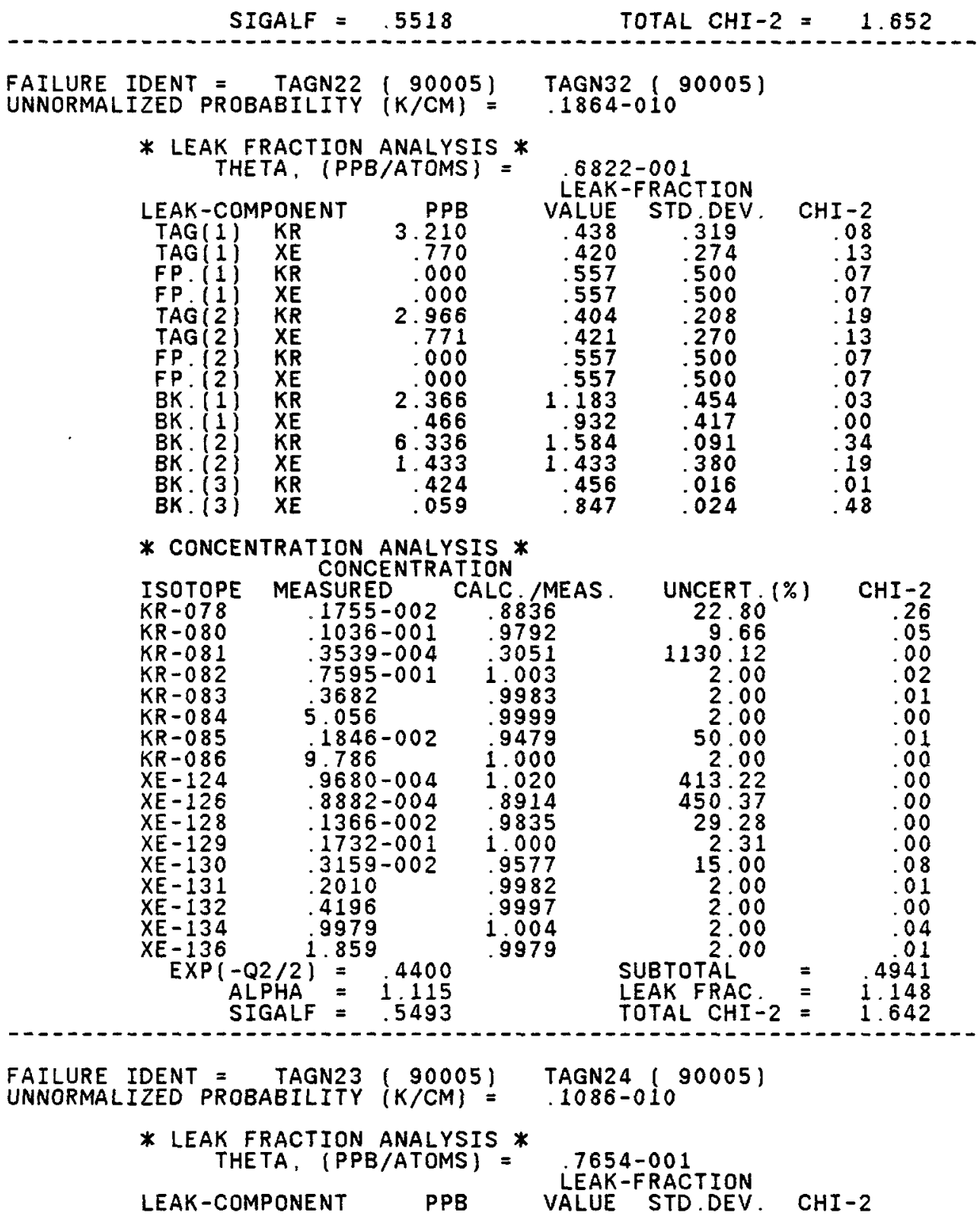




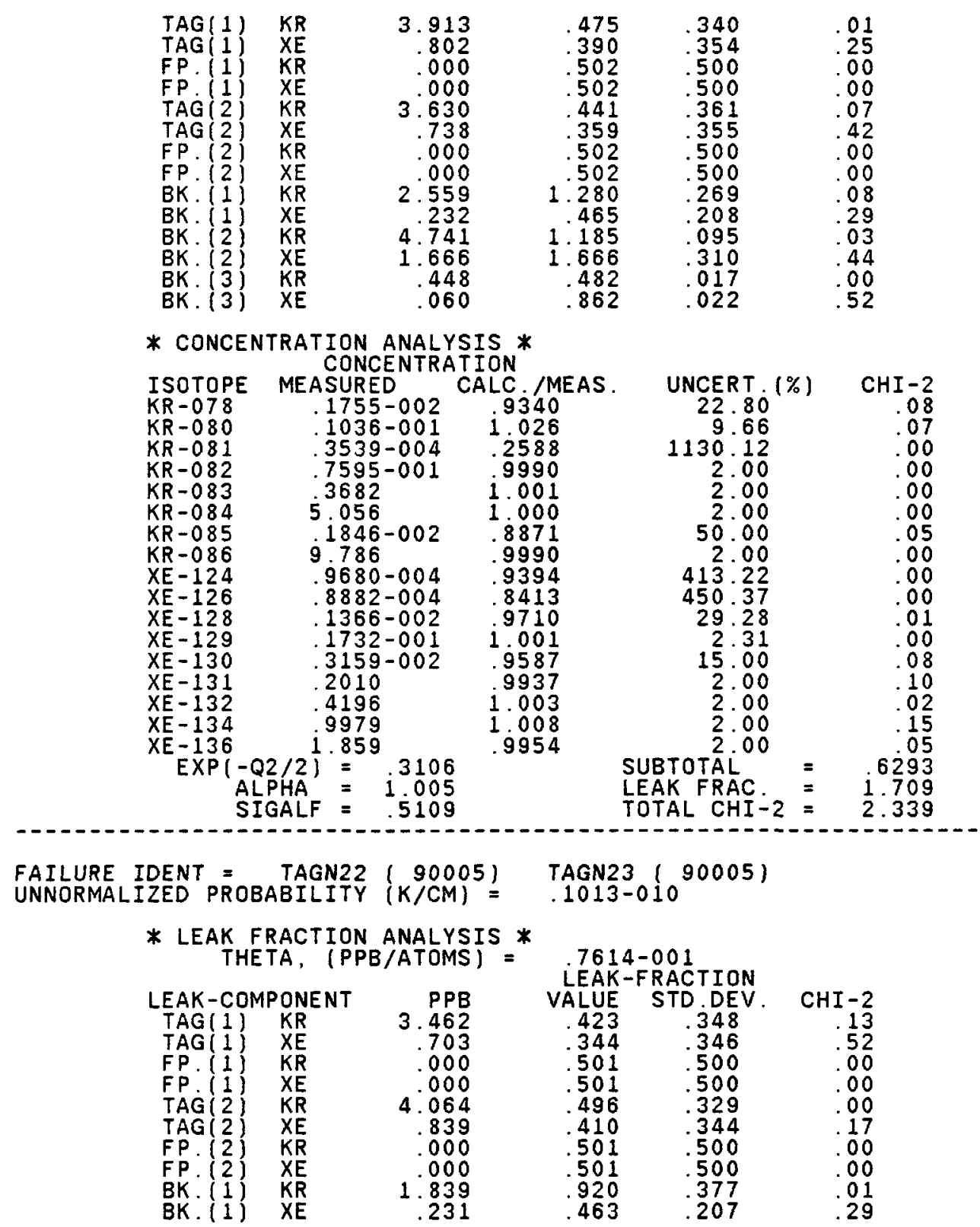




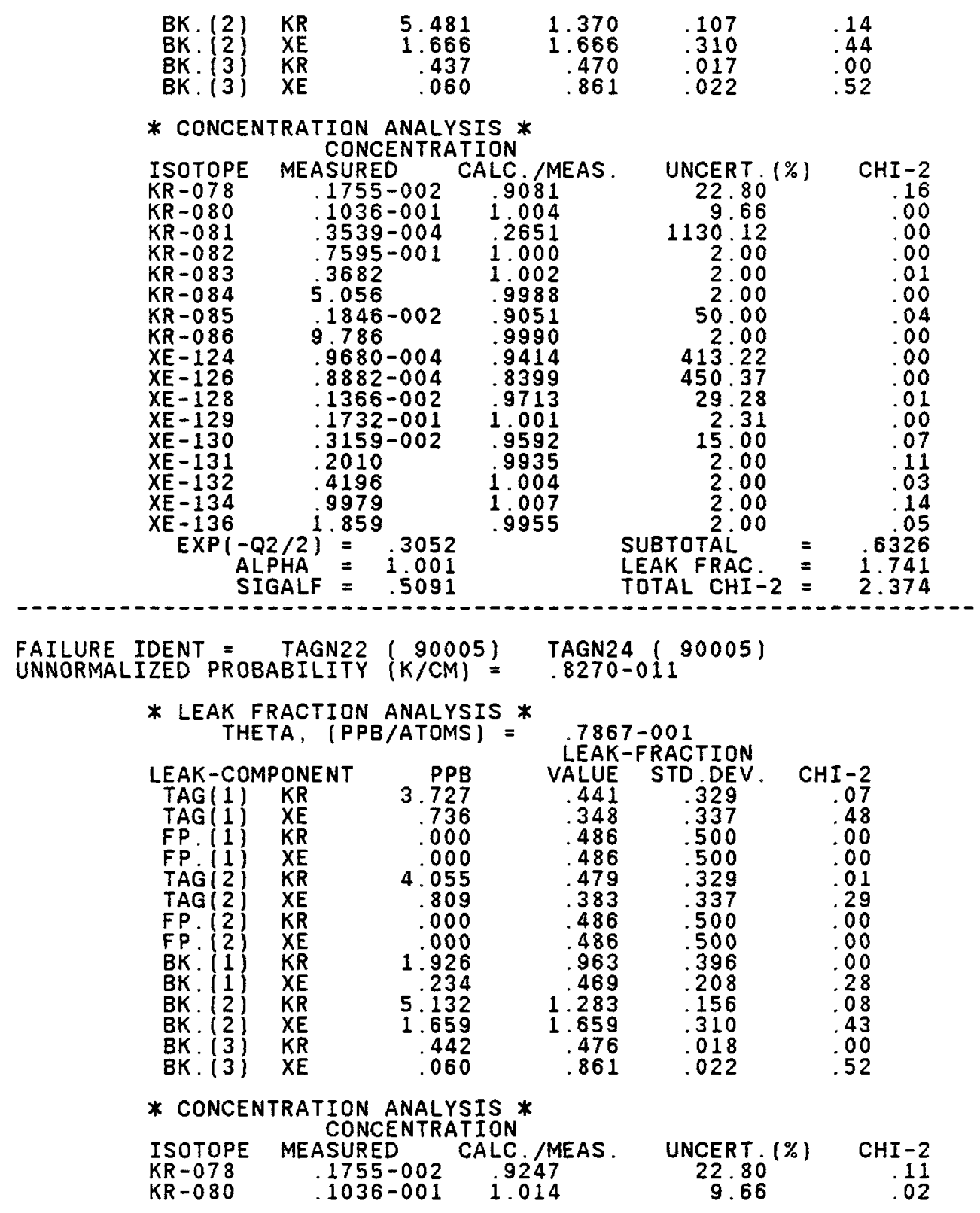


WHC-EP-0190

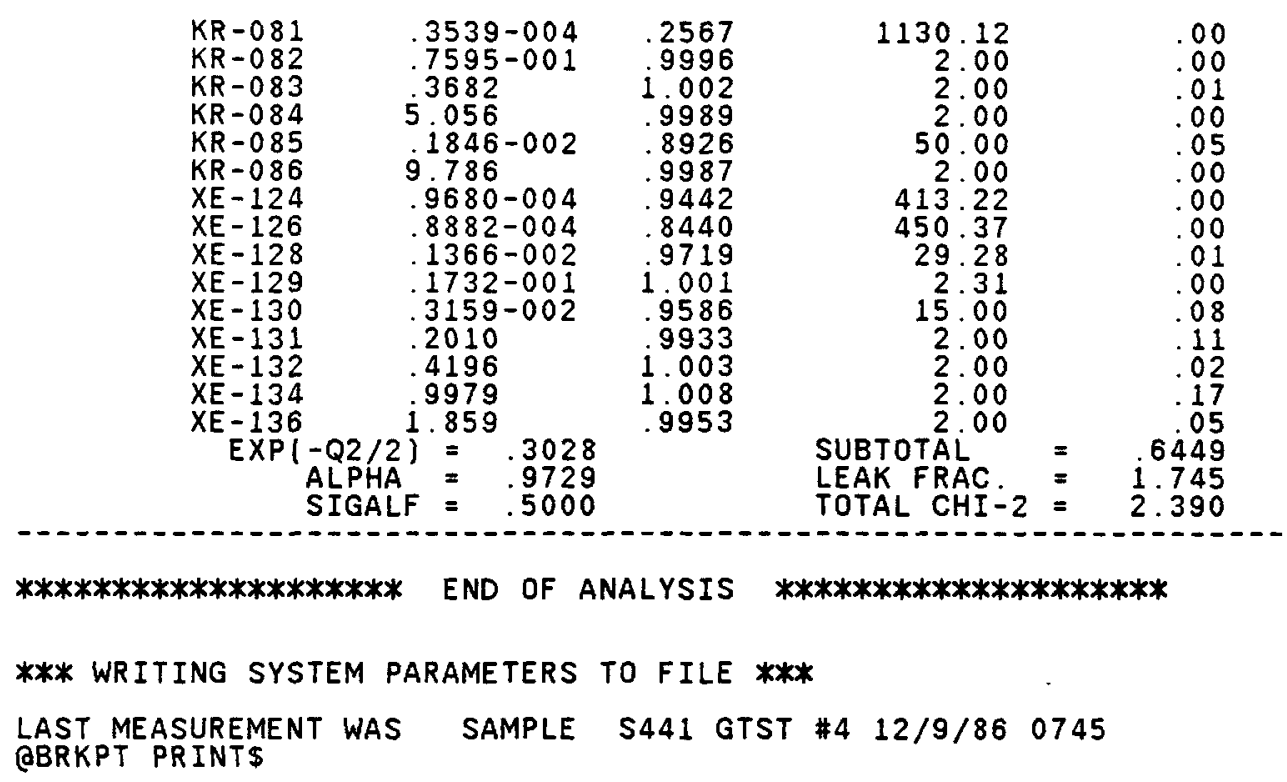


WHC-EP-0190

\section{APPENDIX C}

SEQUENTIAL OUTPUT

QXQT

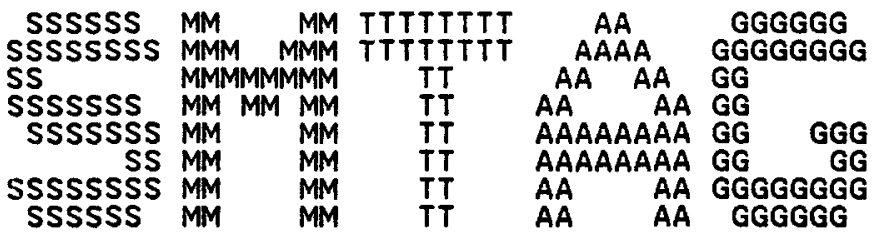

VERSION 2.41 DECEMBER 12,1986

*** READING SYSTEM PARAMETERS FROM FILE ***

LAST MEASUREMENT WAS SAMPLE S441 GTST \#4 12/9/86 0745

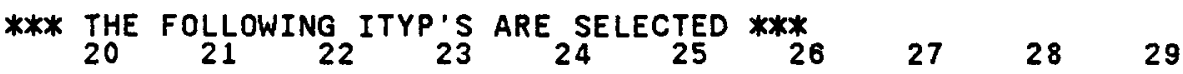

*** READING CONCENTRATION FILE ***

CONF.CONGOI $12 / 02 / 86$

NUMBER OF. TAGS ON CONCENTRATION FILE $=30$
NUMBER OF. TAGS SELECTED BY ITYPE

*** THE DEFAULT ABSOLUTE CONCENTRATION (PPB) UNCERTAINTY WAS RESET:

SIGCO $=.00040$

*** THE FOLLOWING FRACTIONAL CONCENTRATION UNCERTAINTIES WERE RESET: KR-081 2.000 KR-085 $.500 \quad$ XE-130 .150

**** MEASUREMENT ****

SAMPLE S442 GTST \#2 $12 / 10 / 86 \quad 2134$

COVER GAS CONCENTRATION (PPB)

KRYPTON $=130.0 \quad$ XENON $=23.50 \quad$ KRYPTON $/$ XENON $=5.53$

$\begin{array}{lllllll}\text { CONCEN. KR-078 KR-080 KR-081 KR-082 } & \text { KR-083 } & \text { KR-084 }\end{array}$

RELATIVE $\quad .3560-003 \quad .2316-002 \quad .3400-004 \quad .1330-001 \quad .5527-001 \quad 1.000$

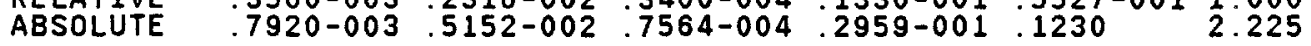

CONCEN. KR-085 KR-086 XE-124 XE-126 XE-128 XE-129

$\begin{array}{lllllll}\text { CONCEN } & \text { KR-085 } & \text { KR-086 } & \text { XE }-124 & \text { XE }-126 & \text { XE -128 } & \text { XE-129 } \\ \text { RELATIVE } & .5530-003 & 57.36 & .2067-001 & .1467-001 & .1091 & 1.000\end{array}$ 
WHC-EP-0190

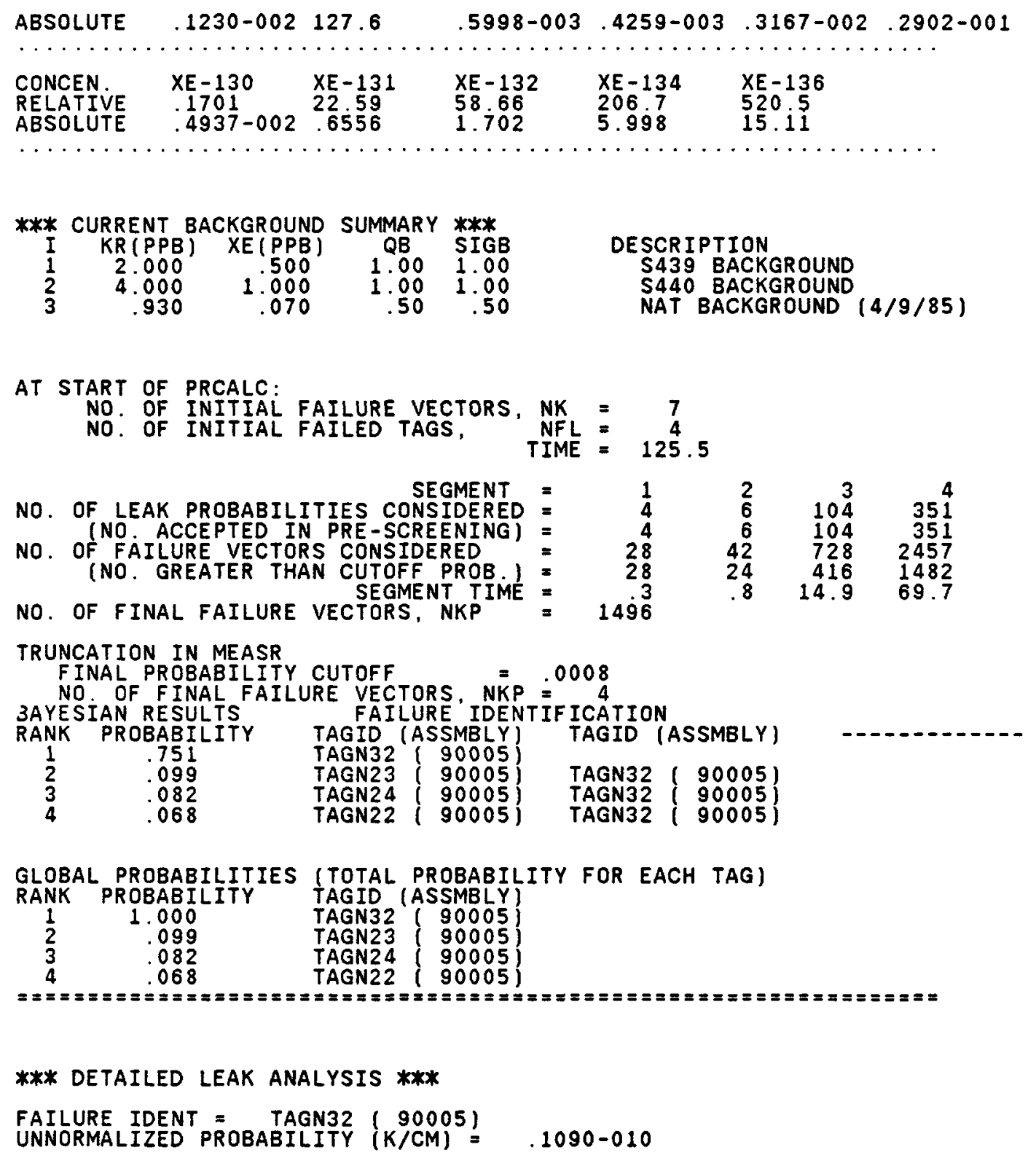




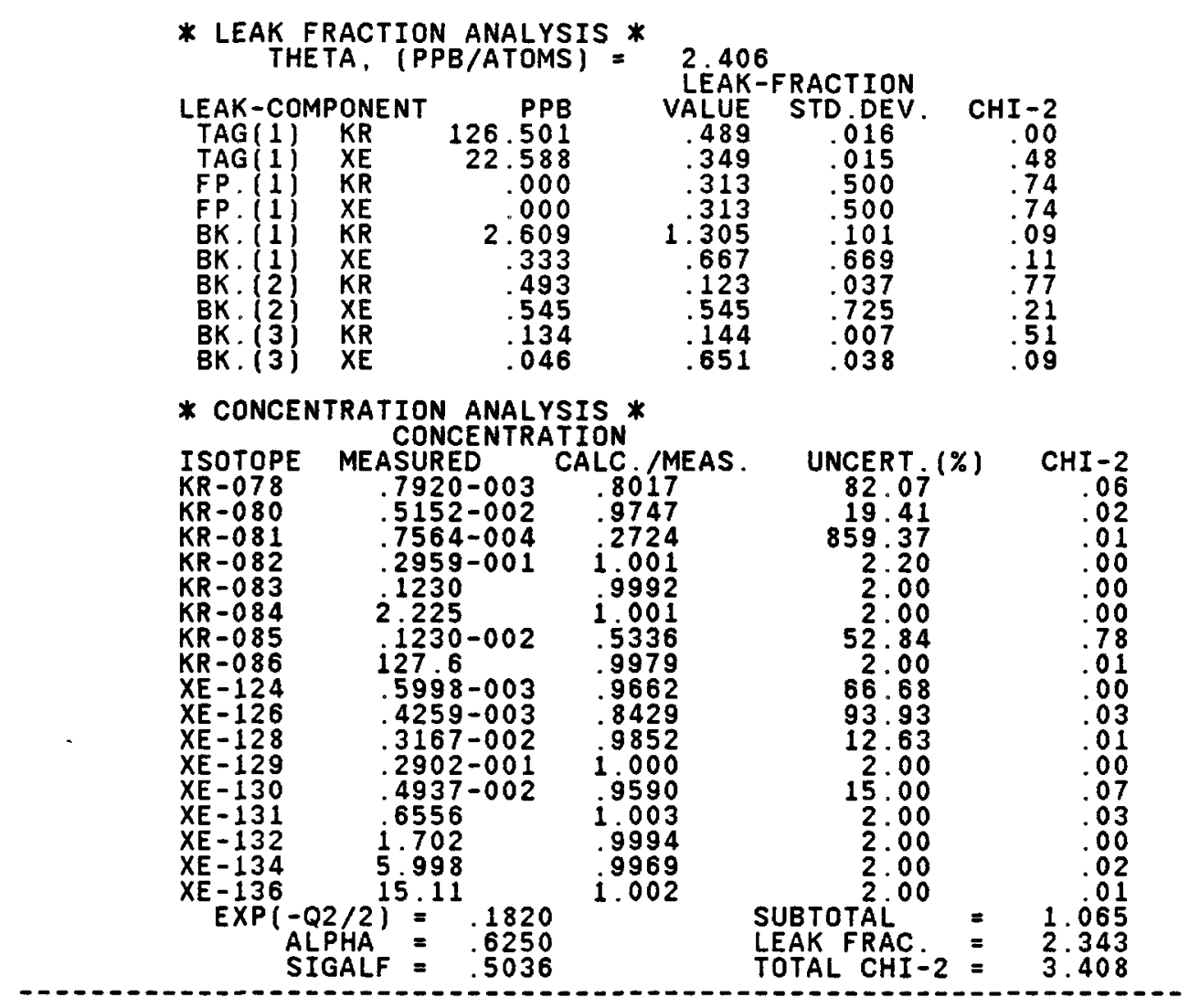

*********************** END OF ANALYSIS $* * * * * * * * * * * * * * * * * * * * * *$ EBRKPT PRINTS 
WHC-EP-0190

\section{DISTRIBUTION}

Number of copies

OFFSITE

2

U.S. Department of Energy -

Headquarters

Facilities, Fuel Cycle, and Test Programs

R. A. Hunter, Director

NE-47

Technology Support Programs

B. J. Rocke, Director

NE-46

2

Argonne National Laboratory East

9700 South Cass Avenue

Argonne, IL 60439

K. Gross

J. B. Lambert

1

Rockwell International

6623 Canoga Ave.

Canoga Park, CA 91303

V. Kesheshian

France

1

PHENIX

J.P. Coulon

Bolte Postale 171

30200 BAGNOLS-sur-CEZE

France

$\underline{\text { Japan }}$

Power Reactor and Nuclear Fuel Development Corporation

2

Hiroshi Hara

SAN-KAI-DOH Building

9-13, 1-Chome, Akasaka,

Minato-Ku, Tokyo, Japan

Toshiba Corporation

2

Tak. Sudo

8 Shinsugita Isogoku Yokohama

Japan

Yuji Sano

4-1, Ukishima-Cho, Kawasaki-Ku,

Kawasaki, 210, Japan 
ONSITE

M. W. Goheen

P7-22

F. E. Hold

P7-22

J. J. McCown

P7-22

3

U.S. Department of Energy -

Richland Operations Office

R. A. Almquist A6-55

Operations Division

J. R. Hunter, Director

A6-55

Nuclear Engineering Program Branch

M. S. Karol, Director

A6-55

DOE Reading Room

A1-65

37

Westinghouse Hanford Company

R. A. Bennett (2)

$\mathrm{N} 2-32$

R. A. Bechtold

N2-04

D. C. Corrigan

N1-70

J. W. Daughtry

HO-35

M. K. Korenko

L5-56

K. D. Dobbin

N3-32

W. D. Leggett

HO-32

R. C. Nichols

B3-02

J. E. Nolan w/o attachment

B3-01

J. E. Pearson

HO-36

R. W. Powell

L5-55

J. A. Rawlins

HO-36

L. R. Rice

R. E. Schenter (2)

L5-57

F. A. Schmittroth (10)

HO-36

A. E. Waltar

J. W. Weber

B. D. Whitten

HO-32

D. W. Wootan

Technical Publications Group (3)

Central Files (2)

Document Clearance (3)

L5-04

HO-36

HO-35

B4-60

L8-15 